\title{
On the hyperspace of subcontinua of a finite graph, II
}

\author{
by \\ R. Dud a (Wrocław)
}

§ 1. Introduction. In the preceding paper [1] we have shown that the hyperspace $C(X)$ consisting of all non-empty subcontinua of a continuum $X$ and metrized by the Hausdorff metric is a polyhedron if and only if $X$ is a finite connected graph. Some results concerning the structure of polyhedra which are hyperspaces have also been proved there, and among them a proposition ([1], corollary 9.2) which can be read as follows: a connected polyhedron $P$ of finite dimension is a hyperspace if and only if its subset $E_{P}$ (whose definition depends on the topology in $P$ only) is homeomorphic to a finite connected graph and $P$ has the structure of the hyperspace for that graph. However, the structure of polyhedra which are hyperspaces has not been discovered so far and it is the aim of the present paper to do it in the particular case of polyhedra which are $C(X)$ for a graph $X$, finite, connected and acyclic (i.e., containing no simple closed curve).

Such graphs are also known under the name of finite dendrites. Thus in the present paper we shall give a Characterization Theorem (see $\S 9$ ) providing in it a complete characterization of those polyhedra which are $C(X)$ for some finite dendrite $X$ (or, what is the same by virtue of theorem 6.4 from [1], which are $C(X)$ for some acyclic continuum $X$ ) and in $\S 10$ we shall provide some examples of such polyhedra.

To gain this aim we shall proceed as follows. We start with a definition, for each finite connected and acyclic graph $X$, of a so called $\alpha$-polyhedron $\alpha(X)$ which we describe as a union. of a certain number of solid triangles and geometric cubes $M_{A C B}$ (see $\S 3$ ). Returning then to a decomposition of the hyperspace $C(X)$ into topological balls $\mathfrak{M}_{A C B}$ (cf. [1], theorem 6.4), we shall define in $\S 6$ and $\S 7$ a homeomorphism

$$
f_{A \subset B}: \mathfrak{M}_{A \subset B} \rightarrow M_{A C B}
$$

for each such $A \subset B$ in a way such that all these homeomorphism can be combined together (see $\S 8$ ) to yield a homeomorphism

$$
f: C(X) \rightarrow \alpha(X)
$$


This shows that $a$-polyhedron $a(X)$ coincides with the hyperspace $C(X)$, whence Characterization Theorem follows easily.

§ 2. Preliminaries. The present paper strictly follows [1] in notions and notations. For the convenience of the reader we shall recall some of them here.

Throughout the paper $X$ is assumed to be a finite connected and acyclic graph, i.e., a connected union of finitely many segments joined by their end-points and containing no simple closed curve. By a segment of $X$ we shall always mean one of those segments, by a subgraph of $X$ a graph contained in $X$ and formed by some of those segments and their end-points, and by a vertex of $X$-an end-point of a segment of $X$. If $v$ is a vertex of $X$ and $\operatorname{ord}_{v} X \geqslant 3$, then $v$ is called a ramification point, and if $\operatorname{ord}_{v} X=1$, then $v$ is called an end-point of $X$. Any subgraph of $X$ which contains no end-point of $X$ is called internal. A segment joining vertex $v$ to vertex $w$ is denoted by $\overline{v w}$.

A topology on $X$ is the identification topology induced by embeddings of segments of $X$ into $X$. Combining some segments into new ones we may assume that each vertex of $X$ is now either an end-point or a ramification point of $X$. Moreover, we assume also that $X$ is metrized by the metric in which each segment of $X$ has length equal to 1 and the distance between any two of its points is equal to the length of the shortest arc joining them. Under these assumptions on the topology and metric in $X$ (equivalent to $(\alpha),(\beta)$ and $(\gamma)$ from [1]) all the results from [1] retain their value here. We shall apply them without further motivation.

Our assumption on $X$ make $X$ very easy to handle. For instance, they give us the following two lemmas:

2.1. Let $A$ be a connected subgraph of $X$. If $A$ is internal and consists of $k$ segments of $X$, then

$$
\operatorname{ord}_{4} X \geqslant k+3 \text {. }
$$

Proof. By hypothesis each vertex $v$ of $A$ is a ramification point of $X$, i.e., of the order $\operatorname{ord}_{v} X \geqslant 3$. Hence if $A$ contains no segment $(k=0)$, i.e., if $A$ is a vertex itself, then clearly $\operatorname{ord}_{A} X \geqslant 3$. Similarly, if $A$ is a segment $(k=1)$, then the two end-points of $A$ are ramification points, and so

$$
\operatorname{ord}_{A} X \geqslant(3-1)+(3-1)=4 \text {. }
$$

Now we shall proceed by induction. Assuming the inductive hypothesis that, for some natural $k \geqslant 0$, if $A$ is a connected and internal subgraph of $X$ consisting of $k$ segments, then $\operatorname{ord}_{A} X \geqslant k+3$, consider a connected and internal subgraph $B$ of $X$ consisting of $k+1$ segments. We
have to show that

$$
\operatorname{ord}_{B} X \geqslant(k+3)+1 \text {. }
$$

For that purpose let $A$ be a connected subgraph of $B$ consisting of $k$ segments and let $B=A \cup \overline{a b}$, where $a \in A$. Since the segment $\overline{a b}$ adds to the order $\operatorname{ord}_{A} X$ at least 1 (because $b$ is a ramification point of $X$ by hypothesis), then it is clear that

$$
\operatorname{ord}_{B} X \geqslant \operatorname{ord}_{A} X+1
$$

whence and from the inductive hypothesis follows (1).

2.2. If $A$ and $B$ are two distinct connected subgraphs. of $X$ such that $A \subset B$ and $B$ is internal, then

$$
\operatorname{ord}_{A} X<\operatorname{ord}_{B} X \text {. }
$$

The proof is by a similarly simple argument.

The notions and symbols not defined in the paper come from [1] and [3].

§ 3. $\alpha$-polyhedron $\alpha(X)$. We shall define here a procedure leading from a finite connected and acyclic graph $X$ to a certain polyhedron which we shall call $\alpha$-polyhedron for $X$ and denote $\alpha(X)$.

Recall that each vertex of $\dot{X}$ is either an end-point or a ramification point (cf. $\S 2$ ), and let $N$ be the number of segments in $X$. By $I^{N}$ we shall mean an $N$-dimensional cube, i.e., a subset of an $N$-dimensional Euclidean space $E^{N}$ consisting of all points $\left(x_{1}, \ldots, x_{N}\right)$ such that $0 \leqslant x_{i} \leqslant 1$ for $i=1, \ldots, N$.

The first and most important (for all that follows depends on it) is an embedding

$$
\text { ı: } X \rightarrow I^{N}
$$

such that each segment of $X$ is identical with a certain edge of $I^{N}$ and that any two distinct segments of $X$ are now perpendicular to each other.

Since $X$ is acyclic by hypothesis, then such an embedding does exist. In general, however, there are many of them and $\iota$ is just a fixed one.

Having defined $\iota$, we attach first to each edge $\iota(B)$ a solid triangle $M_{0 \subset B}$ with the base of length 1 (more precisely, we identify this basewith the edge) in a way such that $M_{0 \subset B}$ is disjoint with $I^{N}$ except for its base and that any two attached triangles $M_{0 C B}$ and $M_{0 \subset B^{\prime}}$ are disjoint except for, perhaps, an end-point $\iota(B) \cap \iota\left(B^{\prime}\right)$ common to their bases $\iota(B)$ and $\iota\left(B^{\prime}\right)$.

Let $\alpha^{\prime}(X)$ denote the union of all $N$ triangles $M_{0 C B}$ attached in that way to $\iota(X)$.

Polyhedron $a(X)$ will be the union of $\alpha^{\prime}(X)$ lying outside $I^{N}$ and of $a^{\prime \prime}(X)$, to the definition of which we now proceed, lying inside $I^{N}$.

If $A$ is an internal connected subgraph of $X$, then by $A^{*}$ we shall mean the middle point of the least face of $I^{N}$ containing all edges of $\iota(A)$ 
As follows by an easy inductive argument, if $A$ consists of $k$ segments, then $A^{*}$ is the middle point of a $k$-dimensional face of $I^{N}$. In particular, if $A$ is a vertex $v$ then $A^{*}=\iota(v)$, if $A$ is a segment then $A^{*}$ is the middle point of the edge $\iota(A)$, etc.

Now let $B$ be a connected subgraph of $X$ such that

$$
A \subset B \subset Q(A, 1),
$$

i.e., let $B$ be a union of $A$ and of some segments of $X$ meeting $A$.

We can then write

(1) $B=A \cup \bigcup_{i=1}^{n} \overline{a_{i} b_{i}}$, where $a_{i} \in A, b_{i} \in B-A$, and all $b_{i}$ are distinct for $i=1,2, \ldots, n$.

By

$$
\left(A^{*} ; \overline{a_{i} b_{i}}\right)
$$

we shall denote the vector with the origin $A^{*}$ and the end-point $\left(A \cup \overline{a_{i} b_{i}}\right)^{*}$, i.e., the vector of length $\frac{1}{2}$, parallel to the edge $\iota\left(\overline{a_{i} b_{i}}\right)$ and joining $A^{*}$ to $\left(A \cup \overline{a_{i} b_{i}}\right)^{*}$ (fig. 1).

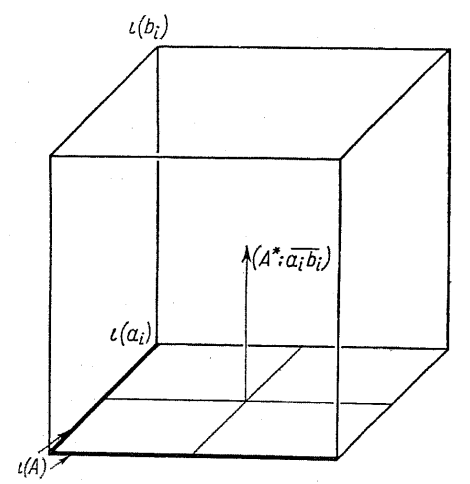

Fig. 1

More generally,

$$
{\underset{i=1}{n}}_{i}^{n}\left(A^{*} ; \overline{a_{i} b_{i}}\right)
$$

will denote the system of $n$ vectors $\left(A^{*} ; \overline{a_{i} b_{i}}\right), i=1,2, \ldots, n$.

We shall regard (2) as a system of coordinates, each vector $\left(A^{*} ; \overline{a_{i} b_{i}}\right)$ being a half-unit vector, and by

$$
M_{A \mathrm{C} B}=\left|{ }_{i=1}^{n}\left(A^{*} ; \overline{a_{i} b_{i}}\right)\right|
$$

we shall mean an $n$-dimensional cube spread over system (2), i.e., the set consisting of all points whose coordinates $\left(y_{1}, \ldots, y_{n}\right)$ in system $(2)$ satisfy conditions $0 \leqslant y_{i} \leqslant \frac{1}{2}$ for $i=1,2, \ldots, n$. Clearly, system (2) of coordinates in $M_{A C B}$ is closely related to the system of coordinates in $I^{N}$ (cf. $\$ 7$ below).

Now we are already able to define $\alpha^{\prime \prime}(X)$. Namely, $\alpha^{\prime \prime}(X)$ is the union of all cubes $M_{A \mathrm{C} B}$, where $A$ runs over all internal connected subgraphs of $X$ and $B=A \cup \bigcup_{i=1}^{n} \overline{a_{i} b_{i}}$ runs over all connected subgraphs of $X$ such that $A \subset B \subset Q(A, 1)$.

In other words,

$$
a(X)=\alpha^{\prime}(X) \cup \alpha^{\prime \prime}(X)=\bigcup_{A \subset B} M_{A \subset B},
$$

where $A \subset B$ runs over all pairs of $X$.

However quite concise, such a definition of $\alpha(X)$ does not make it easy to imagine and so we shall now repeat the last part of it, i.e., the definition of $\alpha^{\prime \prime}(X)$, in a somewhat lengthy but for further investigations more suitable way.

First of all notice that we could restrict ourselves to pairs $A \subset Q(A, 1)$ only, for if $B$ is a subgraph of $X$ such that $A \subset B C Q(A, 1)$ and $B \neq Q(A, 1)$, then the cube $M_{A C B}$ is a face of the cube $M_{B C Q(A, 1)}$.

We shall divide the definition of $\alpha^{\prime \prime}(X)$ into $n+1$ operations

$$
a_{0}, \alpha_{1}, \ldots, \alpha_{n},
$$

where $n$ is the greatest natural number for which there exists an internal subgraph of $X$ consisting of $n$ segments. Operation $a_{k}, k=0,1, \ldots, n$, consists in putting into $I^{N}$ eubes $M_{A \subset Q(A, 1)}$, where $A$ runs over all connected and internal subgraphes of $X$ consisting of $k$ segments precisely.

To construct $a_{0}(X)$ we must then consider all internal vertices of $X$ and for each such a vertex a put into the "corner" of $I^{N}$ determined by $\iota(a)$ the cube $M_{(a) \subset Q(a, 1)}$ of dimension $\operatorname{dim} M_{(a) \subset Q(a, 1)}=\operatorname{ord}_{a} X$ resting upon halves of all the segments of $\iota(X)$ issueing from $\iota(a)$ (see fig. 2).

To construct $a_{1}(X)$ consider the 1 -dimensional faces of $I^{N}$. If a face is the edge $\iota(A)=\bar{\iota}\left(\overline{a) \iota(b)}\right.$ lying in $\iota(X)$, then we put into $I^{N}$ the cube $M_{A C Q(A, 1)}$ resting upon all those edges of the cubes belonging to $a_{0}(X)$, which start from the middle point $A^{*}$ of that face and lie in the 2 -dimensional faces but not in the 1 -dimensional faces of $I^{N}$ (see fig. 3 ).

In fact, if $Q(A, 1)=\bigcup_{i=1}^{n} \overline{a a_{i}} \cup \bigcup_{j=1}^{m} \overline{b b_{j}}$, and

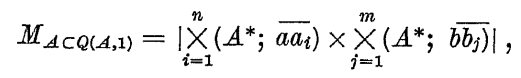




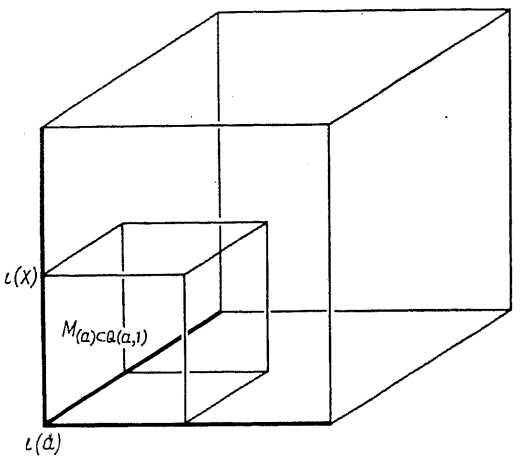

Fig. 2

then the edge $\left(A^{*} ; \overline{a a_{i}}\right)$ belongs to the ball $M_{(a) \subset Q(a, 1)}$ and lies in the 2 -dimensional face of $I^{N}$ determined by the edges $\iota(A)=\iota(\overline{a b})$ and $\iota\left(\overline{a a_{i}}\right)$, and similarly the edge $\left(A^{*} ; b b_{j}\right)$ belongs to the ball $M_{(b) \subset Q(b, 1)}$ and lies in the 2 -dimensional face determined by the edges $\iota(A)=\iota(\overline{a b})$ and $\iota\left(\overline{b b_{j}}\right)$. Other edges are out of question.

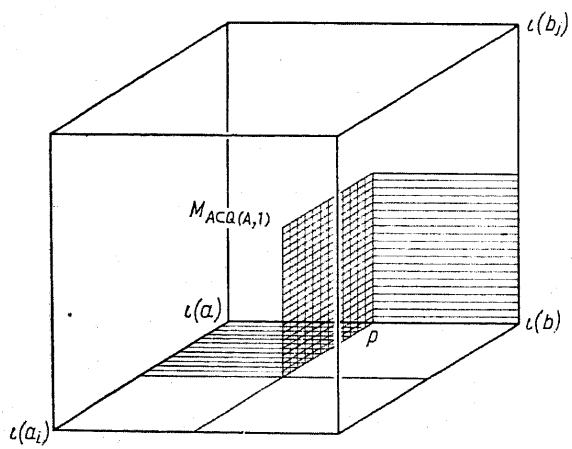

Fig. 3

Now proceed by induction. Suppose that the set of cubes $\alpha_{k-1}(X)$, where $k \geqslant 1$, is already constructed and consider the $k$-dimensional faces of $I^{N}$. If the middle point $p$ of such a face belongs to $\alpha_{k-1}(X)$, then the face is determined by a connected subgraph $A$ of $X$ consisting of $k$ segments of $X$ and $p=A^{*}$.
Two cases are possible:

$1^{\circ}$ If $A$ is internal, then we put into $I^{N}$ the cube $M_{A \subset Q(4,1)}$ resting upon all those edges of the cubes belonging to $\alpha_{k-1}(X)$ which start from the middle point $p=A^{*}$ and lie in the $(k+1)$-dimensional faces but not in the $k$-dimensional faces of $I^{N}$.

In fact, if $p=A^{*}$ and $Q(A, 1)=A \cup \bigcup_{i=1}^{l} \overline{a_{i} b_{i}}$, where $a_{i} \in A$ for $i=1,2, \ldots, l$, then

$$
M_{A \subset Q(A, 1)}=\left|X_{i=1}^{l}\left(A^{*} ; \overline{a_{i} b_{i}}\right)\right|
$$

Edge $\left(A^{*} ; \overline{a_{i} b_{i}}\right)$ lies in the $(k+1)$-dimensional face of $I^{N}$ determined by the $k$-dimensional face, the middle point of which is $p=A^{*}$, and by the edge $\iota\left(\overline{a_{i} b_{i}}\right)$.

Moreover, if $A_{i}$ is a connected subgraph of $A$ consisting of $k-1$ segments and such that $a_{i} \in A_{i}$ (in view of $k \geqslant 1$, subgraph $A_{i}$ clearly exists), then $M_{A_{i} \subset Q\left(A_{i}, 1\right)} \in \alpha_{k-1}(X)$, and the edge $\left(A^{*} ; \overline{a_{i} b_{i}}\right)$ clearly belongs to $M_{A_{i} \subset Q\left(A_{i}, 1\right)}$.

Other edges are out of question.

$2^{\circ}$ If $A$ is not internal, we leave it as it is.

(Note, that by 2.1 each cube of the set $\alpha_{k}(X)$ has dimension not less than $k+3$, and by 2.2 this dimension grows up from step to step.)

Having defined $\alpha_{k}(X)$ we proceed to $a_{k+1}(X)$, and so on until we come to the maximal $n$ for which there exists an internal subgraph of $X$ con sisting of $n$ segments. Naturally, this is an end of our construction, and so

$$
\alpha^{\prime \prime}(X)=\alpha_{0}(X) \cup \alpha_{1}(X) \cup \ldots \cup \alpha_{n}(X)
$$

This completes our (second) definition of the $\alpha$-polyhedron $\alpha(X)$.

It is not difficult to prove that a connected polyhedron $\alpha(X)$ is a topological invariant of $X$. However, we do not need to bother about that at the moment, because it will be a simple consequence of theorem 8.2 (to the proof of which we shall proceed) stating that if a polyhedron $P$ is the hyperspace $C(X)$ for some finite connected and acyclic graph $X$, then $P$ coincides with the $\alpha$-polyhedron $\alpha(X)$.

§ 4. Geometric diagram of the polyhedron $a(X)$. Polyhedron $a(X)$, as defined in $\S 3$ for a finite, connected and acyclic graph $X$, has rather complicated structure and in general it is of quite a large dimension (it can be easily shown that dimension of $\alpha(X)$ equals to the number of end-points of $X$ ). In $\S 9$ we shall prove that polyhedron $\alpha(X)$ coincides with the hyperspace $C(X)$. In view then of a great importance of $a$-polyhedra (at least for the present paper) it may be, perhaps, of some value to describe here a certain way of looking upon $\alpha$-polyhedron 
through its geometric diagram in 3-dimensional Euclidean space $E^{3}$, and so we shall describe here such a diagram shortly. However, these diagrams will not be used until the last $\S 10$.

In construction of $\alpha$-polyhedron $\alpha(X)$ we have started with the solid triangles $M_{0 C B}$. The set of all those triangles,

$$
\alpha^{\prime}(X)=\bigcup_{B} M_{0 \subset B},
$$

where $B$ runs over all segments of $X$, can be represented in $E^{3}$ in the following way (see fig. 4): first we embed $X$ into the plane $O x y$ in a way such that each segment of $X$ is a straight-line segment of length 1 (it is possible because $X$ is a graph both finite and acyclic) and then upon each segment $B=\overline{a b}$ of this homeomorph of $X$ we erect the isosceles perpendicular triangle of height $\frac{1}{2}$. In fig. 4 this triangle is denoted by $\widetilde{m a b}$.

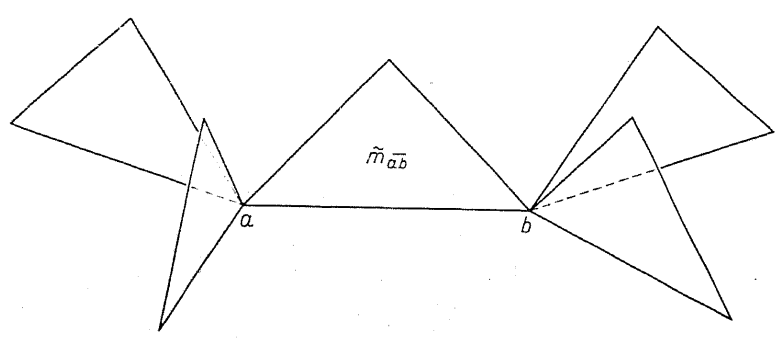

Fig. 4

Clearly, $M_{0 \subset \overline{a b}}=\tilde{m}_{\overline{a b}}$. Let us agree that under this homeomorphism the segment $\iota \overline{(a) \iota(b)}$, which is a base of the triangle $M_{0<\overline{a b}}$, goes onto the two upper edges of the triangle $\tilde{m} \bar{a} \bar{b}$ in such a way that the half-segment $\overline{\iota(a)(\overline{a b})^{*}}$ goes linearly onto the edge joining $a$ to the top vertex, and the half-segment $\left(\overline{a b)^{*} \iota(b)}\right.$ goes linearly onto the edge joining the top vertex to $b$. Hence, in particular, the top vertex of $\tilde{m}_{\overline{a b}}$ corresponds to the joint $(a b)^{*}$ of $M_{0} \overline{a b}$.

This way of looking upon $\alpha^{\prime}(X)$ can be extended first to a geometric diagram of the set

$$
\alpha^{\prime}(X) \cup a_{0}(X)=\bigcup_{B} M_{0 \subset B} \cup \bigcup_{a} M_{(a) \subset Q(a, 1)},
$$

where $a$ runs over all internal vertices of $X$.

Indeed, if $a$ is a vertex (but not an end-point) of $X$ and

$$
Q(a, 1)=\bigcup_{i=1}^{n} \overline{a a_{i}}
$$

then we erect upon $a$ a perpendicular straight-line segment of length 1 , and treating this segment as a base, we build upon it $n$ isosceles triangles, each of height $\frac{1}{2}$ and each lying over one of the segments issueing from $a$.

This bunch, which we denote by $m_{a}$ (see fig. 5 ), represents the $n$-dimensional cube

$$
M M_{(a) \subset Q(a, 1)}=\left|{\underset{i=1}{n}}_{i=1}^{n}\left((a)^{*} ; \overline{a a_{i}}\right)\right| \text {. }
$$

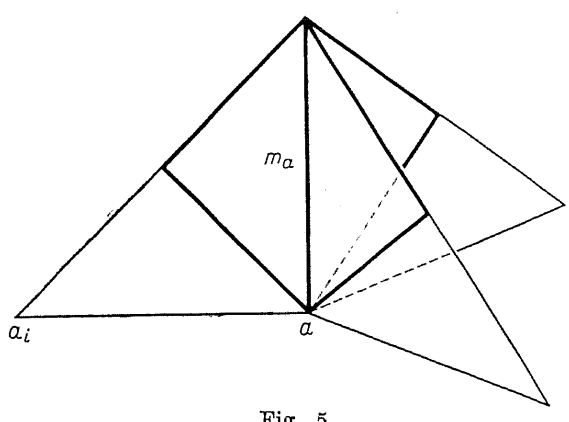

Fig. 5

Let us agree that the point $a$ of $m_{a}$ represents the point $(a)^{*}=\iota(a)$ of that cube, that the lower side of the triangle lying over segment $\overline{a a_{i}}$ represents all points of that cube whose only non-zero coordinate corresponds to the vector $\left((a)^{*} ; \overline{a a}_{i}\right)$ and that this correspondence is linear. Since the vector $\left((a)^{*} ; \overline{a a_{i}}\right)$ is a vector with the origin $\iota(a)$ and the endpoint $\left(\overline{a a_{i}}\right)^{*}$, that lower side can be identified with the corresponding side of the triangle $\tilde{m}_{\overline{a a_{i}}}$. Furthermore, let us also agree that the upper side of the triangle of $m_{a}$ lying over $\overline{a a_{i}}$ represents all points of our cube ||$_{i=1}^{n}\left((a)^{*} ; \overline{a a_{i}}\right) \mid$ whose coordinate in system $\underset{i=1}{x}\left((a)^{*} ; \overline{a a_{i}}\right)$ corresponding to the vector $\left((a)^{*} ; \overline{a a_{i}}\right)$ is equal to $\frac{1}{2}$. Such an upper side represents then actually an $(n-1)$-dimensional face of our cube.

Now we shall extend the geometric diagram of $\alpha^{\prime}(X) \cup \alpha_{0}(X)$ to a geometric diagram of $\alpha^{\prime}(X) \cup a_{0}(X) \cup a_{1}(X)$ by defining it for each ball $M_{A \subset Q(A, 1)} \in a_{1}(X)$.

Let then $A=\overline{a b}$, where $\operatorname{ord}_{a} X=n+1$ and $\operatorname{ord}_{b} X=m+1$. As follows from the definition of the diagram $\alpha^{\prime}(X) \cup \alpha_{0}(X)$, the bunches $m_{a}$ and $m_{b}$ meet at a point representing $(\overline{a b})^{*}$ and joining the upper sides of the two triangles, one of $m_{a}$ and the other of $m_{b}$, and both lying over $\overline{a b}$. These sides correspond, respectively, to an $n$-dimensional face of the $(n+1)$-dimensional cube $M_{(a) \subset Q(a, 1)}$ representing certain points of that 
cube and to an $m$-dimensional face of the $(m+1)$-dimensional cube representing certains points of $M_{(b) \subset Q(b, 1)}$.

Upon these two faces we construct the square $m_{\overline{a b}}$ representing the $(n+m)$-dimensional cube $M_{\bar{a} \bar{b} c Q(\overline{a b}, 1)}$ in a way such that its lower'sides are identical with the sides of the corresponding triangles of $m_{a}$ and $m_{b}$ and represent the same continua (see fig. 6).

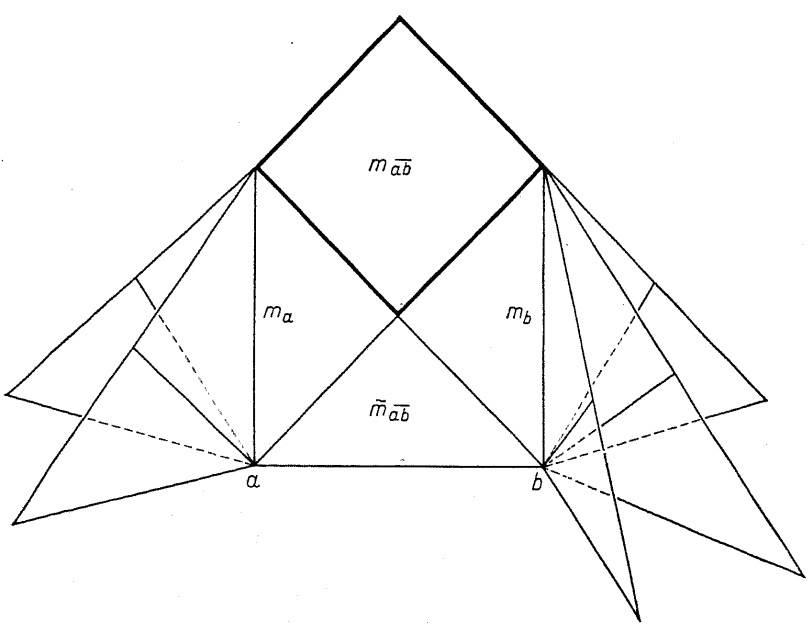

Fig. 6

Our way of looking upon $\alpha^{\prime}(X) \cup a_{0}(X) \cup a_{1}(X)$ through its geometric diagram lying in $E^{3}$ is convenient for many purposes, but there are several obstacles to its extension to a similar diagram of the set $\alpha^{\prime}(X) \cup a_{0}(X) \cup$ $\cup a_{1}(X) \cup a_{2}(X)$ to say nothing of the whole polyhedron $a(X)$. The main cause is the complex structure of the polyhedron involved. Nevertheless, a rather natural extension of the geometric diagram of $\alpha^{\prime}(X) \cup a_{0}(X) \cup$ $\checkmark a_{1}(X)$ to a geometric diagram of the whole $a(X)$ does exist and gives some idea on the composition of this polyhedron. We shall now describe it shortly.

A geometric diagram of $\alpha(X)$ will consist of the diagram of $\alpha^{\prime}(X)$ and diagrams $m_{A}$ of all sets $M_{A \subset Q(A, 1)}$, where $A$ runs over all connected and internal subgraphs of $X$.

In order to define a diagram of $\dot{M}_{A C Q(A, 1)}$ we must first distinguish in $A$ a subgraph $A_{0}$ which we shall call a middle element of $A$. Let $\delta(A)=k$, If $k=2 l$ (resp., $k=2 l+1$ ), then $A_{0}$ is a vertex (resp., a segment) of $A$ such that $A-A_{0}$ has at least two components of diameter $l$ each. For $k=0$ we put $A_{0}=A$. Since $\delta(A)=k$ implies that $A$ contains an are $I$ of length $k$ consisting of $k$ segments and $k+1$ joining them vertices (and does not contain any are of length $k+1$ ), then $A_{0}$ can be also defined as the middle vertex of $L$ if $k=2 l$ or the middle segment of $L$ if $k=2 l+1$. It is clear that $A_{0}$ does not depend on $L$ (for otherwise we would obtain a contradiction of $\delta(A)=k$ ) and so the middle element of $A$ is unique.

Consider now two cases:

I. If $A_{0}$ is a segment, then we define $m_{A}$ to be a square of side $\sqrt{2}$, lying just over $A_{0}$ at a distance (measured from $A_{0}$ to the lower vertex of the square) equal to $k / 2$ (see fig. 7 ).

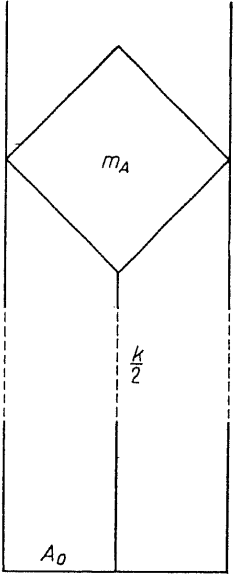

Fig. 7

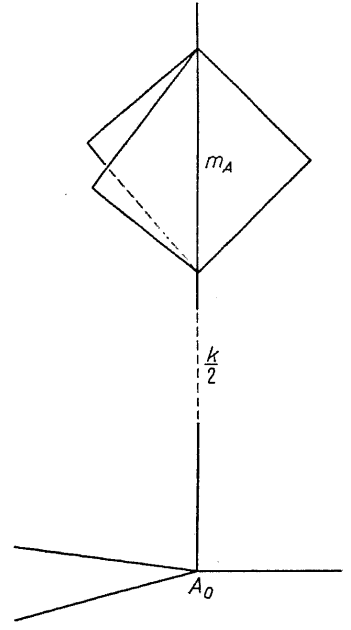

Fig. 8
II. And if $A_{0}$ is a vertex, then we define $m_{A}$ to be a bunch of $n$ triangles, where $n=\operatorname{ord}_{A} X$ if $l=0$, and $n$ is the number of all internal ares in $X$ issueing from $A_{0}$ and disjoint outside $A_{0}$ if $l>0$. The triangles of the bunch are all isosceles of height $\frac{1}{2}$ and their common base is a straightline segment of length 1 lying just over $A_{0}$ at a distance (measured from $A_{0}$ to its lower end) equal to $k / 2$. Each triangle lies over half of the first segment of one of those $n$ arcs in $A$ (see fig. 8).

It should be clear that the geometric diagram of $\alpha(X)$, which we have just in that way defined, embraces the diagram of $\alpha^{\prime}(X) \cup \alpha_{0}(X) \cup a_{1}(X)$ and so that it is an extension of that diagram to a diagram of the whole polyhedron $\alpha(X)$. 
In comparison with the diagram of $\alpha^{\prime}(X) \cup \alpha_{0}(X) \cup \alpha_{1}(X)$ the diagram of the whole $\alpha(X)$ has some faults. It may happen that $m_{A}=m_{A^{\prime}}$ in spite of $A \neq A^{\prime}$, and thus a diagram $m_{A}$ of some $M_{A C Q(A, 1)}$ actually often represents a whole system of cubes $M_{A^{\prime} \subset Q\left(A^{\prime}, 1\right)}$. Also some of $m_{A}$ and $m_{A^{\prime}}$ can meet although cubes $M_{A C Q(A, 1)}$ and $M_{A^{\prime} C Q\left(A^{\prime}, 1\right)}$ are disjoint.

However, it can be said to its advantage that if $m_{A}$ and $m_{A^{\prime}}$ are disjoint, then are also disjoint $M_{A C Q(A, 1)}$ and $M_{\mathcal{A}^{\prime} \subset Q\left(A^{\prime}, 1\right)}$. Moreover, the geometric diagram of $\alpha(X)$ is defined uniquely and corresponds to one $X$ only.

§5. Families $\mathfrak{M}_{A C B}$. Let us recall that two connected subgraphs, $A \subset B$, of a finite connected graph $X$ are called a pair of $X$ if $A=0$ and $B$ is a segment of $X$ or if $A$ is internal and $B$ satisfies the condition $A \subset B C$ $C Q(A, 1)$ (i.e., $B$ is a union of $A$ and of some segments of $X$ meeting $A$ ).

To each pair $A \subset B$ corresponds a certain family $\mathfrak{M}_{A \subset B}$ of subcontinua of $X$. In the considered in the present paper case of acyclic $X$, the family $\mathfrak{M}_{\triangle \subset B}$ can be defined as follows (cf. $[1], \S 5$ ):

$$
\mathfrak{M}_{A \subset B}=\{C \in C(X): A \subset C \subset B\} .
$$

Occasionally, however, we shall also deal with the families $\mathfrak{M}_{A \subset B}$, where $A$ and $B$ are two subggraphs of $X$ such that $A \subset B$, but not necessarily forming a pair.

5.1. If $A \subset B$ and $A^{\prime} \subset B^{\prime}$ are two pairs, then

(1) $\mathfrak{M}_{A \subset B} \cap \mathfrak{M}_{A^{\prime} \subset B^{\prime}} \neq 0$ if and only if $A \cup A^{\prime} \subset B \cap B^{\prime} \neq 0$,

and if, moreover, $A \cup A^{\prime} C B \cap B^{\prime} \neq 0$, then

(2) $\mathfrak{M}_{A \subset B} \cap \mathfrak{M}_{A^{\prime} C B^{\prime}}=\left\{C \in C(X): A \cup A^{\prime} C C \subset B \cap B^{\prime}\right\}$.

Proof. If

(3)

$$
\mathfrak{M}_{A \subset B} \cap \mathfrak{M}_{A^{\prime} \subset B^{\prime}} \neq 0,
$$

then there exists a continuum $C$ such that

$$
\text { Hence }
$$

$$
A \subset C \subset B \text { and } A^{\prime} \subset C \subset B^{\prime} \text {. }
$$

(5)

$$
A \cup A^{\prime} \subset C \subset B \cap B^{\prime}
$$

and so

$$
A \cup A^{\prime} \subset B \cap B^{\prime} \neq 0 \text {. }
$$

Conversely, if (6) holds, then $C=B \cap B^{\prime}$ is a continuum and since by (6) it satisfies (5) and, consequently, (4), then (3) also holds.

Thus we have proved (1).

Equality (2) easily follows from the equivalence of (4) and (5).

5.2. If $A \subset B$ and $A^{\prime} \subset B^{\prime}$ are two pairs such that $A \neq 0 \neq A^{\prime}$ and

$$
A \cup A^{\prime} \subset B \cap B^{\prime},
$$

$$
\begin{gathered}
\varrho^{1}\left(A, A^{\prime}\right) \leqslant 1, \\
\varrho^{1}\left(A \cup A^{\prime}, B \cap B^{\prime}\right) \leqslant 1 .
\end{gathered}
$$

If, moreover, $A \cap A^{\prime}=0$, then there exist vertices $a, a^{\prime} \in X$ such that

$$
A=(a), \quad A^{\prime}=\left(a^{\prime}\right) \quad \text { and } \quad B \cap B^{\prime}=\overline{a a^{\prime}} \subset X .
$$

Proof. We first prove (8). In fact, since, by hypothesis, $B \subset Q(A, 1)$ and $B^{\prime} \subset Q\left(A^{\prime}, 1\right)$, then (7) implies

$$
A \cup A^{\prime} \subset Q(A, 1) \cap Q\left(A^{\prime}, 1\right) .
$$

Hence, in particular, $A \subset Q\left(A^{\prime}, 1\right)$ and $A^{\prime} \subset Q(A, 1)$, i.e., $\varrho^{\mathbb{1}}\left(A, A^{\prime}\right) \leqslant 1$. To prove (9) observe that

$$
\begin{aligned}
\varrho^{1}\left(A \cup A^{\prime}, B \cap B^{\prime}\right) & =\sup _{y \in B \cap B^{\prime}} \varrho\left(A \cup A^{\prime}, y\right) \\
& =\min \left[\sup _{y \in B \cap B^{\prime}} \varrho(A, y), \sup _{y \in B \cap B^{\prime}} \varrho\left(A^{\prime}, y\right)\right] \\
& \leqslant \min \left[\sup _{y \in \bar{B}} \varrho(A, y), \sup _{y \in B^{\prime}} \varrho\left(A^{\prime}, y\right)\right] \\
& \leqslant \min \left[\varrho^{1}(A, B), \varrho^{1}\left(A^{\prime}, B^{\prime}\right)\right] \leqslant 1,
\end{aligned}
$$

where the first equality follows from (7).

Finally, if $A \cap A^{\prime}=0$, then by (8) there must exist vertices $a, a^{\prime} \in X$ such that $A=(a), A^{\prime}=\left(a^{\prime}\right)$ and in $X$ there is a segment $\overline{a a^{\prime}} \subset X$. In view of (7), the common part $B \cap B^{\prime}$ must then be non-empty and so $B \cap B^{\prime}$ is a connected subgraph. By (7) and (9) we must have $B \cap B^{\prime}=\overline{a a^{\prime}}$.

5.3. If $A \subset B$ and $A^{\prime} \subset B^{\prime}$ are two pairs such that $A_{0}=A \cap A^{\prime} \neq 0$, then $B \cap B^{\prime} \subset Q\left(A_{0}, 1\right)$.

In fact, let $x \in B \cap B^{\prime}$. If there is no segment of $B \cap B^{\prime}$ containing $x$, then $x$ must be a vertex and, in view of the acyclicity of $B \cap B^{\prime}$ following from that of $X$, we must also have $(x)=B \cap B^{\prime}$. But then, as follows from $A \cap A^{\prime} \subset B \cap B^{\prime}$, we must have $(x)=A \cap A^{\prime}=A_{0}$, whence, a fortiori, $x \in Q\left(A_{0}, 1\right)$. Therefore, let $\overline{a a^{\prime}}$ be a segment of $X$ such that $x \in \overline{a a^{\prime}} C B \cap B^{\prime}$. Since $B \subset Q(A, 1)$ and $B^{\prime} \subset Q\left(A^{\prime}, 1\right)$, one of the endpoints $a$ and $a^{\prime}$ of $\overline{a a^{\prime}}$ must belong to $A$ and the other to $A^{\prime}$. Let $a \in A$ and $a^{\prime} \in A^{\prime}$. If $a \in A-A^{\prime}$ and $a^{\prime} \in A^{\prime}-A$, then, by the acyclicity of $X$, we would also have $A \cap A^{\prime}=0$ which is impossible. Hence either $a \in A \cap A^{\prime}$ or $a^{\prime} \in A \cap A^{\prime}$, and in both cases $x \in Q\left(A_{0}, 1\right)$. 
§ 6. Homeomorphisms $f_{A C B}$ for $A \subset B$ running over all pairs of $X$ with $A=0$. In the preceding paper [1] we have shown that each family $\mathfrak{M}_{A C B}$, where $A \subset B$ is any pair of a finite and connected graph $X$, is a topological ball ([1], lemma 5.2) and that, in the considered here case of acyclic $X$, their union yields polyhedron $C(X)$ as a cellular complex ([1], theorem 6.3)

$$
C(X)=\bigcup_{A \subset B} \mathfrak{M}_{A \subset B}
$$

In $\S 3$ of the present paper we have defined, for each finite connected and acyclic graph $X$, polyhedron $\alpha(X)$ as a union of geometric triangles and cubes $M_{A \subset B}$, where $A \subset B$ runs again over all pairs of $X$,

$$
\alpha(X)=\bigcup_{A \subset B} M_{A C B} .
$$

Now we proceed to show that both these sets, $C(X)$ and $\alpha(X)$, are homeomorphic to each other. With that end in mind, assume that there is given, as defined in $\S 3$, an embedding

$$
\text { ı: } X \rightarrow E^{N}
$$

and that $a$-polyhedron $\alpha(X)$ based upon this embedding is already constructed.

In this $\S 6$ we shall define homeomorphisms

$$
f_{A \subset B}: \mathfrak{M}_{A \subset B} \rightarrow M_{A C B}
$$

for all pairs $A \subset B$ of $X$ with $A=0$, in the next $\S 7$ such homeomorphisms will be defined for all other pairs of $X$, and in $\S 8$ we shall show that all these homeomorphisms $f_{\triangle C B}$ can be combined together to yield a homeomorphism

$$
f: C(X) \rightarrow a(X) .
$$

In order to define homeomorphism (1) for a pair $A \subset B$ with $A=0$ we shall first define homeomorphism

$$
f_{A \subset B}^{\prime}: \mathfrak{M}_{A C B} \rightarrow E^{2},
$$

then define a homeomorphism

$$
f_{A \subset B}^{\prime \prime}: f_{A \subset B}^{\prime}\left(\mathfrak{M}_{A \subset B}\right) \rightarrow M_{A \subset B},
$$

and finally, put $f_{A C B}=f_{A C B}^{\prime \prime} f_{A \subset B}^{\prime}$.

Let $B=\overline{a b}$. Each continuum $C \subset B$ is then uniquely determined by the pair of real numbers $\left(r\left(m_{C}\right), \frac{1}{2} \delta(C)\right)$, where $m_{C}$ is the middle point of $C$ and $r\left(m_{C}\right)$ is the real number denoting the position of the point $m_{C}$ in $\overline{a b}\left(r(a)=0 \leqslant r\left(m_{C}\right) \leqslant 1=r(b)\right)$.
Clearly, the correspondence $C \rightarrow\left(r\left(m_{C}\right), \frac{1}{2} \delta(C)\right)$ between continua $C C B$ and pairs $(x, y)$ of real numbers from the triangle of vertices $(0,0)$, $(0,1)$ and $\left(\frac{1}{2}, \frac{1}{2}\right)$ is one-to-one and continuous.

Put

$$
f_{A \subset B}^{\prime}(C)=\left(r\left(m_{C}\right), \frac{1}{2} \delta(C)\right) .
$$

Hence $f_{A C B}^{\prime}\left(\mathfrak{M}_{A C B}\right)$ is a triangle whose base represents the points of $B$, one side-the subcontinua of $B$ containing $a$, and the other sidethose containing $b$. The top vertex represents $B$ itself (see fig. 9).

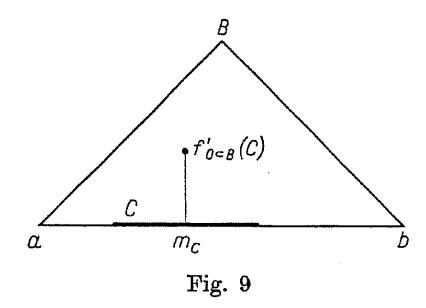

Now turn to the edge $\iota(B) \subset I^{N}$ and define $f_{A \subset B}^{\prime \prime}$ as a homeomorphism which maps $f_{A C B}^{\prime}\left(\mathfrak{M l}_{A \subset B}\right)$ onto the triangle $M_{A C B}$ in a way such that the side $\overline{(a) B}$ goes linearly onto the half-segment $\overline{\iota(a) B^{*}}$, and the side $\overline{B(b)}$ goes linearly onto the half-segment $\overline{B^{*} \iota(b)}$.

It is clear that

6.1. If $B$ is a segment of $X$, then the mapping $f_{0 C B}: \mathfrak{M}_{0 C B} \rightarrow M_{0 \subset B}$ is a homeomorphism onto.

6.2. If $a$ is a vertex of $X$ and $B$ any segment of $X$ containing a, then $f_{0 C B}(a)=\iota(a)$.

6.3. If $B$ and $B^{\prime}$ are two distinct segments of $X$, then $f_{0 \subset B}\left(\mathfrak{M}_{0 \subset B}\right)$ and $f_{0 \subset B^{\prime}}\left(\mathfrak{M}_{0 \subset B^{\prime}}\right)$ are disjoint outside $I^{N}$.

§ 7. Homeorphisms $f_{A C B}$ for $A \subset B$ running over all pairs of $X$ with $A \neq 0$. Let $A \subset B$ be a pair of $X$ such that $A \neq 0$. We can then write $B$ in the form

(1) $B=A \cup \bigcup_{i=1}^{i} \overline{a_{i} b_{i}}$, where $a_{i} \in A, b_{i} \in B-A$, and all $b_{i}$ are distinct for $i=1,2, \ldots, n$.

With each such a pair there is connected system of $n$ vectors

$$
{\underset{i=1}{n}}_{i}^{n}\left(A^{*} ; \overline{a_{i} b_{i}}\right)
$$


which can be regarded as a system of coordinates, each vector $\left(A^{*} ; \overline{a_{i} b_{i}}\right)$ being a half-unit vector (cf. §3).

The cube

$$
M_{A \subset B}=\left|X_{i=1}^{n}\left(A^{*} ; \overline{a_{i} b_{i}}\right)\right|
$$

spread over system (2) is contained in $I^{N}$ and the system of coordinates (2) is closely related to that in $I^{N}$.

$$
\text { In fact, let }
$$

$$
i_{1}, \ldots, i_{p}
$$

be the numbers of all those axes of $I^{N}$ which are parallel each to a certain edge of $I^{N}$ contained in $\iota(A)$.

Similarly, let

$$
j_{1}, \ldots, j_{r}
$$

be the numbers of all those axes of $I^{N}$ which are parallel each to a certain edge contained in $\iota(\overline{B-A})$.

By the supposition that any two distinct edges of $\iota(X)$ are perpendicular to each other, sequences (4) and (5) are disjoint.

Now, each point $p=\left(t_{1}, \ldots, t_{n}\right)$ of $(3)$ is also a point of $I^{N}$. Let $\left(x_{1}, \ldots, x_{N}\right)$ be the system of its coordinates in $I^{N}$. It should be clear that these two systems are connected by the relations

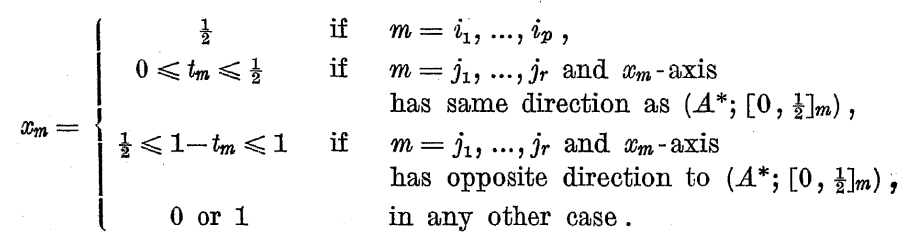

The symbol $\left(A^{*} ;\left[0, \frac{1}{2}\right]_{m}\right)$ denotes here the half-unit vector $\left(A^{*} ; \overline{a_{i} b_{i}}\right)$ parallel to the $x_{m}$-axis.

Now we proceed to the definition of the homeomorphism

$$
f_{A C B}: \mathfrak{M}_{A C B} \rightarrow M_{A C B} \text {. }
$$

Namely, if $O \in \mathfrak{M}_{A \subset B}$, then $C$ may be expressed in the form

$$
C=A \cup \bigcup_{i=1}^{n} \overline{a_{i} b_{i}}\left(t_{i}\right)
$$

where $0 \leqslant t_{i} \leqslant 1$ and $\overline{a_{i} b_{i}}\left(t_{i}\right)$ denotes a subsegment of $\overline{a_{i} b_{i}}$ containing $a_{i}$ and having length $t_{i}, i=1,2, \ldots, n$.
We define

$$
f_{A C B}(C)=\left(\frac{1}{2} t_{1}, \ldots, \frac{1}{2} t_{n}\right),
$$

where $\left(\frac{1}{2} t_{1}, \ldots, \frac{1}{2} t_{n}\right)$ are coordinates in system (2), and so obtain a map $f_{A \subset B}: \mathfrak{M}_{A \subset B} \rightarrow E^{N}$. The range of this map is equal to $M_{A \subset B}$ and so it is quite obvious that

7.1. If $A \subset B$ is a pair with $A \neq 0$ and $B$ satisfies (1), then the mapping

$$
f_{A \subset B}: \mathfrak{M}_{A C B} \rightarrow M_{A C B}
$$

is a homeomorphism onto.

Since $M_{A \subset B}$ is an $n$-dimensional cube and $n=\operatorname{ord}_{A} B$, we infer that $\therefore \quad$ 7.2. If $A \subset B$ is a pair with $A \neq 0$ and $B$ satisfies (1), then $\operatorname{dim} \mathfrak{M}_{A \subset B}$ $=\operatorname{ord}_{A} B$. In particular, if $B=Q(A, 1)$, then $\operatorname{dim} \mathfrak{M}_{A \subset B}=\operatorname{ord}_{A} X$.

It is also clear from the definition of the map $f_{A \subset B}$ that

7.3. If $A \subset B$ is a pair with $A \neq 0$ and if $S$ is a subgraph of $X$ such that $A \subset S \subset B$, then $f_{A \subset B}(S)=S^{*}$.

7.4. If $A \subset B$ is a pair with $A \neq 0$ and if $A$ is a vertex and $S$ a segment such that $A \in S \subset B$, then $f_{A \subset B} \mid \mathfrak{M}_{A \subset S}$ is a linear homeomorphism mapping $\mathfrak{M}_{A \subset S}$ onto half-edge $\overline{A^{*} S^{*}}$ in such a way that $f_{A \subset B}(A)=A^{*}$ and $f_{A \subset B}(S)=S^{*}$.

$\therefore$ Now we shall prove the sequence of lemmas 7.5-7.9 which are needed to prove in $\S 8$ the consistency of homeomorphisms $f_{A C B}$.

7.5. Let $A$ be a non-empty and connected subgraph of $X$. If $A \subset B$ and $G \subset H$ are pairs such that

$$
A \subset G \subset H \subset B,
$$

then

and

(8) $\quad f_{A C B}\left(\mathfrak{M}_{G \subset H}\right)=f_{G \subset H}\left(\mathfrak{M}_{G \subset H}\right)$ is a face of the eube $M_{A C B}$.

Proof. By virtue of (6) the family $\mathfrak{M}_{G \subset H}$ is contained in the family $\mathfrak{M}_{A \subset B}$ and so we have to show that if $C \in \mathfrak{M}_{G \subset H}$, then $f_{G \subset H}(C)=f_{A \subset B}(C)$.

If $G=H=S$, then $\mathfrak{M}_{G C H}=(S)$ and, by proposition $7.3, f_{G C H}(S)$ $=S^{*}=f_{A C B}(S)$. Hence we may assume that $G \neq H$.

Let $B$ satisfy (1). The subgraphs $G$ and $H$ are then the unions of $A$ and of some segments $\overline{a_{i} b_{i}}, i=1,2, \ldots, n$. Denoting an empty set by $\overline{a_{0} b_{0}}$ and reordering, if necessary, the segments $\overline{a_{1} b_{1}}, \ldots, \overline{a_{n} b_{n}}$, we may then write

$$
G=A \cup \bigcup_{i=0}^{k} \overline{a_{i} b_{i}} \quad \text { and } \quad H=A \cup \bigcup_{i=0}^{l} \overline{a_{i} b_{i}},
$$

where $0 \leqslant k<l \leqslant n$.

Fundamenta Mathematicae, T. LXIII 
By virtue of (3) and proposition 7.1 we then have

$f_{A \subset B}: \mathfrak{M}_{A \subset B} \rightarrow||_{i=1}^{n}\left(A^{*} ; \overline{\left.a_{i} b_{i}\right)} \mid\right.$ and $\quad f_{G \subset H}: \mathfrak{M}_{G \subset H} \rightarrow\left|\underset{i=k+1}{l}\left(G^{*} ; \overline{a_{i} b_{i}}\right)\right|$.

As is easy to see, $G^{*}$ is a point of $\left|X_{i=1}^{n}\left(A^{*} ; \overline{a_{i} b_{i}}\right)\right|$ of the coordinates $\left(t_{1}, \ldots, t_{n}\right)$ (in system (2)) such that

$$
t_{1}=\ldots=t_{k}=\frac{1}{2}, \quad t_{k+1}=\ldots=t_{n}=0,
$$

and the cube $\left|\underset{i=k+1}{x}\left(G^{*} ; \overline{a_{i} b_{i}}\right)\right|$ is a face of the cube $\left|x_{i=1}^{n}\left(A^{*} ; \overline{a_{i} b_{i}}\right)\right|$ consisting of all points of the coordinates $\left(u_{1}, \ldots, u_{n}\right)$ (in the same system) such that

(9) $\quad u_{1}=\ldots=u_{k}=\frac{1}{2}, \quad 0 \leqslant u_{i} \leqslant \frac{1}{2}$ for $i=k+1, \ldots, l$,

$$
u_{l+1}=\ldots=u_{n}=0 \text {, }
$$

or, in the system $\underset{i=k+1}{\stackrel{x}{X}}\left(G^{*} ; a_{i} b_{i}\right)$, of the coordinates $u_{k+1}, \ldots, u_{l}$ such that

$$
0 \leqslant u_{i} \leqslant \frac{1}{2} \quad \text { for } \quad i=k+1, \ldots, l .
$$

Moreover, points (9) and (10) are identical, i.e., they are the same points of $I^{N}$.

Now, if $C \in \mathfrak{M}_{G C H}$, then $C=A \cup \bigcup_{i=1}^{n} \overline{a_{i} b_{i}\left(z_{i}\right)}$, where $z_{1}=\ldots=z_{k}=1$, $0 \leqslant z_{i} \leqslant 1$ for $i=k+1, \ldots, l$, and $z_{l+1}^{\prime}=\ldots=z_{n}=0$, and so, by the definitions of $f_{A C B}$ and $f_{G C H}$,

Hence

$$
f_{A \subset B}(C)=\left(\frac{z_{1}}{2}, \ldots, \frac{z_{n}}{2}\right) \quad \text { and } \quad f_{G \subset H}^{m}(C)=\left(\frac{z_{k+1}}{2}, \ldots, \frac{z_{l}}{2}\right) .
$$

$$
f_{A C B}(O)=f_{G \subset H}(C) \text {. }
$$

7.6. If $A \subset B$ and $A^{\prime} \subset B^{\prime}$ are two pairs such that $A \neq 0 \neq A^{\prime}$ and $A \cup A^{\prime} \subset B \cap B^{\prime}$, then

$$
f_{A C B} \mid \mathfrak{M}_{A \cup A^{\prime} C B \cap B^{\prime}}=f_{A^{\prime} C B^{\prime}} \mathfrak{M}_{\overline{A \cup A^{\prime} C B \cap B^{\prime}}}
$$

Proof. If $A \cap A^{\prime}=0$, then, by lemma 5.4, there exist vertices $a, a^{\prime} \in X$ such that $A=(a), A^{\prime}=\left(a^{\prime}\right)$, and $B \cap B^{\prime}=\overline{a a^{\prime}} \subset X$. Therefore, if $O \in \mathfrak{M}_{A \cup A^{\prime} C B \cap B^{\prime}}$, then $a \cup a^{\prime} \subset C \subset \overline{a a^{\prime}}$, and so $C=\overline{a a^{\prime}}$. Hence, by lemma $7.3, f_{A C B}(C)=\left(\overline{a a^{\prime}}\right)^{*}=f_{A^{\prime} \subset B^{\prime}}(C)$.

Finally, if $A \cap A^{\prime} \neq 0$, then $A \cup A^{\prime}$ is a connected subgraph of $X$ and, by lemma 5.2, $A \cup A^{\prime} \subset B \cap B^{\prime}$ is a pair. Since

$$
A \subset A \cup A^{\prime} \subset B \cap B^{\prime} \subset B
$$

then, by lemma 7.5 ,

and, similarly

$$
f_{A \subset B} \mid M_{A \cup A^{\prime} C B \cap B^{\prime}}=f_{A \cup A^{\prime} C B \cap B^{\prime}},
$$

$$
f_{A^{\prime} \subset B^{\prime}} \mid M_{A \cup A^{\prime} \subset B \cap B^{\prime}}=f_{A \cup A^{\prime} \subset B \cap B^{\prime}} .
$$

The two equalities imply (11).

7.7. If $A \subset B$ and $A^{\prime} \subset B^{\prime}$ are two pairs such that

then

$$
A \cup A^{\prime} \subset B \cap B^{\prime} \quad \text { and } \quad A_{0}=A \cap A^{\prime} \neq 0,
$$

is a face of each of the following three cubes $M_{A \subset B}, M_{A^{\prime} \subset B^{\prime}}$ and $M_{A_{0} \subset Q\left(A_{0}, 1\right)}$. Indeed, equality (13) follows from lemma 7.6.

From (12) and lemma 5.3 we infer that

$$
A_{0} \subset A \cup A^{\prime} \subset B \cap B^{\prime} \subset Q\left(A_{0}, 1\right),
$$

and the following two sequences of inclusions are obvious

$$
\begin{aligned}
& A \subset A \cup A^{\prime} \subset B \cap B^{\prime} \subset B, \\
& A^{\prime} \subset A \cup A^{\prime} \subset B \cap B^{\prime} \subset B^{\prime} .
\end{aligned}
$$

Now it suffices to apply in each case (8) of lemma 7.5.

7.8. If $A \subset B$ and $A^{\prime} \subset B^{\prime}$ are two pairs such that $A \neq 0 \neq A^{\prime}$, then

$$
f_{A \subset B}\left(\mathfrak{M}_{\triangle \subset B}\right) \cap f_{A^{\prime} \subset B^{\prime}}\left(\mathfrak{M}_{A^{\prime} \subset B^{\prime}}\right) \neq 0
$$

if and only if $A \cup A^{\prime} C B \cap B^{\prime}$.

If, moreover, $A \cup A^{\prime} C B \cap B^{\prime}$, then

$f_{A \subset B}\left(\mathfrak{M}_{A \subset B}\right) \cap f_{A^{\prime} \subset B^{\prime}}\left(\mathfrak{M}_{A^{\prime} \subset B^{\prime}}\right)=f_{A \subset B}\left(\mathfrak{M}_{A \cup A^{\prime} \subset B \cap B^{\prime}}\right)=f_{A^{\prime} \subset B^{\prime}}\left(\mathfrak{M}_{A \cup A^{\prime} \subset B \cap B^{\prime}}\right)$.

Proof. Let $A^{*}=\left(x_{1}, \ldots, x_{N}\right)$ and $A^{\prime *}=\left(x_{1}^{\prime}, \ldots, x_{N}^{\prime}\right)$. By the definition of $A^{*}$ (cf. $\S 3$ ),

$$
x_{i}=\left\{\begin{array}{cl}
\frac{1}{2} & \text { if } \iota(A) \text { contains an edge parallel to the } x_{i} \text {-axis, } \\
0 \text { or } 1 & \text { otherwise, }
\end{array}\right.
$$

and, similarly,

$$
x_{j}^{\prime}=\left\{\begin{array}{cl}
\frac{1}{2} & \text { if } \iota\left(A^{\prime}\right) \text { contains an edge parallel to the } x_{j}^{\prime} \text {-axis, } \\
0 \text { or } 1 & \text { otherwise. }
\end{array}\right.
$$

Let $i_{1}, \ldots, i_{k_{1}}$


be the sequence of all indices $i_{r}$ for which $x_{i_{r}}=\frac{1}{2}$, and let

$$
j_{1}, \ldots, j_{l_{1}}
$$

be the sequence of all indices $j_{s}$ for which $x_{j_{s}}^{\prime}=\frac{1}{2}$.

Similarly, let

$$
i_{k_{1}+1}, \ldots, i_{k_{2}}
$$

be the sequence of all indices $i_{r}$ such that the $x_{i_{r}}$-axis is parallel to a certain segment of $\iota(\overline{B-A})$, and

$$
j_{l_{1}+1}, \ldots, j_{l_{2}}
$$

be the sequence of all indices $j_{s}$ such that the $x_{j_{s}}^{\prime}$-axis is parallel to a certain segment of $\iota \overline{\left(B^{\prime}-A^{\prime}\right)}$.

By the definition of a map $f_{A \subset B}$, the set $f_{A \subset B}\left(\mathfrak{M}_{A \subset B}\right)$ consists of all points $\left(t_{1}, \ldots, t_{N}\right) \in I^{N}$ such that

$$
t_{m}=\left\{\begin{array}{cl}
\frac{1}{2} \quad \text { for } m=i_{1}, \ldots, i_{k_{1}}, \\
0 \leqslant t_{m} \leqslant \frac{1}{2} \text { or } \frac{1}{2} \leqslant t_{m} \leqslant 1 \text { for } m=i_{k_{1}+1}, \ldots, i_{k_{2}}, \\
0 \text { or } 1 \quad \text { otherwise }
\end{array}\right.
$$
that

And the set $f_{A^{\prime} \subset B^{\prime}}\left(\mathfrak{M}_{A^{\prime} \subset B^{\prime}}\right)$ consists of all points $\left(t_{1}^{\prime}, \ldots, t_{N}^{\prime}\right) \in I^{N}$ such

$$
t_{m}^{\prime}=\left\{\begin{array}{cl}
\frac{1}{2} \quad \text { for } \quad m=j_{1}, \ldots, j_{l_{1}}, \\
0 \leqslant t_{m}^{\prime} \leqslant \frac{1}{2} \text { or } \frac{1}{2} \leqslant t_{m}^{\prime} \leqslant 1 \text { for } m=j_{l_{1}+1}, \ldots, j_{l_{\mathbf{a}}}, \\
0 \text { or } 1 \quad \text { otherwise }
\end{array}\right.
$$

Let $\left(t_{1}, \ldots, t_{N}\right) \in f_{A \subset B}\left(\mathfrak{M}_{A \subset B}\right)$ and $\left(t_{1}^{\prime}, \ldots, t_{N}^{\prime}\right) \in f_{A^{\prime} \subset B^{\prime}}\left(\mathfrak{M}_{A^{\prime} \subset B^{\prime}}\right)$, and suppose that

$$
\left(t_{1}, \ldots, t_{N}\right)=\left(t_{1}^{\prime}, \ldots, t_{N}^{\prime}\right)
$$

Two cases are now to be examined.

I. $A \cap A^{\prime}=0$. In this case sequences (14) and (15) are disjoint and so if (18) holds, then we must have $k_{1}=l_{1}=0$, i.e., both $A$ and $A^{\prime}$ must be vertices. Since they are distinct vertices, say $A=(a)$ and $A^{\prime}=\left(a^{\prime}\right)$, then there exists an axis, let it be the $m_{0}$-axis, such that the $m_{0}$ th coordinate of $\iota(a)$ is 0 and the $m_{0}$ th coordinate of $\iota\left(a^{\prime}\right)$ is 1 (or the other way round). Now if $\varrho\left(a, a^{\prime}\right) \geqslant 2$, then also the sequences (16) and (17) would be disjoint, $m_{0}$ could belong to one of the sequences (16) and (17) only, and so the equality (18) could not hold. Hence there exists a segment $\overline{a a^{\prime}} \subset X$. Its image $\iota\left(\overline{a a^{\prime}}\right)$ is parallel to the $m_{0}$-axis and so if $m_{0}$ belongs to (16), then the $m_{0}$ th coordinate of a point $\left(t_{1}, \ldots, t_{N}\right)$ of $f_{A \subset B}\left(\mathfrak{M}_{A \subset B}\right)$ can assume any value $0 \leqslant t_{m_{0}} \leqslant \frac{1}{2}$, and similarly, if $m_{c}$ belongs to (17) then the $m_{0}$ th coordinate $t_{m_{0}}^{\prime}$ of a point $\left(t_{1}^{\prime}, \ldots, t_{N}^{\prime}\right) \in f_{A^{\prime} \subset B^{\prime}}\left(\mathfrak{M}_{\mathcal{A}^{\prime} \subset B^{\prime}}\right)$ satisfies $\frac{1}{2} \leqslant t_{m_{0}}^{\prime} \leqslant 1$. Therefore $(18)$ can hold if and only if $m_{0}$ belongs both to sequence (16) and to sequence (17) (i.e., if and only if $a \cup a^{\prime}$ $\left.C B \cap B^{\prime}\right)$ and in that case $t_{m_{0}}=t_{m_{0}}^{\prime}=\frac{1}{2}$. And since (16) and (17) are disjoint except for $m_{0}$ (because $X$ is acyclic by hypothesis), then the only common point of $f_{A \subset B}\left(\mathfrak{M}_{A \subset B}\right)$ and $f_{A^{\prime} C B^{\prime}}\left(\mathfrak{M}_{A^{\prime} \subset B^{\prime}}\right)$ can be the one whose coordinates $t_{m}$ are all equal either to 0 or to 1 except for $t_{m_{0}}=\frac{1}{2}$, i.e., the point $\left.\overline{\left(a a^{\prime}\right.}\right)^{*}$.

However, by lemma $5.2, B \cap B^{\prime}=\overline{a a^{\prime}}$ and so $\left.\mathfrak{M}_{A \cup A^{\prime} \subset B \cap B^{\prime}}=\overline{\left(a a^{\prime}\right.}\right)$. Hence and from lemma 7.3 we infer that

$$
\begin{aligned}
f_{A \subset B}\left(M_{A C B}\right) \cap f_{A^{\prime} \subset B^{\prime}}( & \left.M_{A^{\prime} \subset B^{\prime}}\right)=\left(\overline{a a^{\prime}}\right)^{*} \\
& =f_{A \subset B}\left(\mathfrak{M}_{A \cup A^{\prime} \subset B \cap B^{\prime}}\right)=f_{A^{\prime} \subset B^{\prime}}\left(\mathfrak{M}_{A \cup A^{\prime} \subset B \cap B^{\prime}}\right) .
\end{aligned}
$$

II. $A \cap A^{\prime} \neq 0$. In this case, if $m$ belongs to (16) and (17) simultaneously, then the projections of $A^{*}$ and $A^{\prime *}$ onto the $x_{m}$-axis are the same point, and so for both $t_{m}$ and $t_{m}^{\prime}$ we have either $0 \leqslant t_{m} \leqslant \frac{1}{2}$ and $0 \leqslant t_{m}^{\prime} \leqslant \frac{1}{2}$ or $\frac{1}{2} \leqslant t_{m} \leqslant 1$ and $\frac{1}{2} \leqslant t_{m}^{\prime} \leqslant 1$. Hence, if (18) holds, we must have

$$
t_{m}=t_{m}^{\prime}=\left\{\begin{array}{cl}
\frac{1}{2} \quad & \text { for } \quad m \epsilon\left(i_{1}, \ldots, i_{k_{1}}\right) \cup\left(j_{1}, \ldots, j_{l_{1}}\right) \\
0 \leqslant t_{m}= & t_{m}^{\prime} \leqslant \frac{1}{2} \text { or } \frac{1}{2} \leqslant t_{m}=t_{m}^{\prime} \leqslant 1 \\
& \text { for } m \epsilon\left(i_{k_{1}+1}, \ldots, i_{k_{2}}\right) \cap\left(j_{l_{1}+1}, \ldots, j_{l_{2}}\right) \\
0 \text { or } 1 & \text { otherwise }
\end{array}\right.
$$

In other words, point (18) belongs to $f_{A \cup A^{\prime} C B \cap B^{\prime}}\left(\mathfrak{M}_{A \cup A^{\prime} C B \cap B^{\prime}}\right)$ and this is possible if and only if $\left(i_{1}, \ldots, i_{k_{1}}\right) \subset\left(j_{1}, \ldots, j_{l_{2}}\right)$ and $\left(j_{1}, \ldots, j_{l_{1}}\right)$ $\subset\left(i_{k_{1}}, \ldots, i_{k_{2}}\right)$, i.e., if and only if $A \subset B^{\prime}$ and $A^{\prime} \subset B$, which is obviously equivalent to $A \cup A^{\prime} \subset B \cap B^{\prime}$.

A trivial consequence of lemmas $7.8,7.7$ and 7.1 is the following: proposition

7.9. Let $A \subset B$ and $A^{\prime} \subset B^{\prime}$ be two pairs of $X$ such that both $A$ and $A^{\prime}$ are non-empty. If the eubes $M_{A \subset B}$ and $M_{A^{\prime} \subset B^{\prime}}$ meet together, then their common part is their common face.

§ 8. Consistency of homeomorphisms $f_{A C B}$. The basic result here is the following proposition, which proves that all homeomorphisms $f_{A \subset B}$, where $A \subset B$ runs over all pairs of $X$, can be combined to yield a homeomorphism from $C(X)$ onto $\alpha(X)$ (cf. 8.2 below).

8.1. If $A \subset B$ and $A^{\prime} \subset B^{\prime}$ are two pairs of $X$, then

(i) $\quad f_{A C B}\left|\mathfrak{M}_{A C B} \cap \mathfrak{M}_{A^{\prime} C B^{\prime}}=f_{A^{\prime} \subset B^{\prime}}\right| \mathfrak{M}_{A \subset B} \cap \mathfrak{M}_{A^{\prime} C B^{\prime}}$,

(ii) $f_{A C B}\left(\mathfrak{M}_{A C B} \cap \mathfrak{M}_{A^{\prime} C B^{\prime}}\right)$

$$
=f_{A C B}\left(\mathfrak{M}_{A \subset B}\right) \frown f_{A^{\prime} \subset B^{\prime}}\left(\mathfrak{M}_{A^{\prime} \subset B^{\prime}}\right)=f_{A^{\prime} \subset B^{\prime}}\left(\mathfrak{M}_{A \subset B} \cap \mathfrak{M}_{A^{\prime} \subset B^{\prime}}\right) .
$$


Proof. I. We first show that (i) and (ii) are fulfilled if both $A$ and $A^{\prime}$ are empty, $A=0=A^{\prime}$.

Indeed, by virtue of lemma 6.3 , if $B$ and $B^{\prime}$ are distinct segments of $X$, then

$$
f_{0 C B}\left(\mathfrak{M}_{0 \subset B}\right) \cap f_{0 C B^{\prime}}\left(\mathfrak{M}_{0 \subset B^{\prime}}\right)=f_{0 \subset B}\left(\mathfrak{M}_{0 \subset B}\right) \cap f_{0 \subset B^{\prime}}\left(\mathfrak{M}_{0 \subset B^{\prime}}\right) \frown I^{N} .
$$

But by the definition of the map $f_{0 c B}$ we have

and, similarly,

$$
f_{0 \subset B}\left(\mathfrak{M}_{0 \subset B}\right) \cap I^{N}=\iota(B),
$$

Hence

$$
f_{0 \subset B^{\prime}}\left(\mathfrak{M}_{0 \subset B^{\prime}}\right) \cap I^{N}=\iota\left(B^{\prime}\right) .
$$

$$
f_{0 C B}\left(\mathfrak{M}_{0 \subset B}\right) \cap f_{0 \subset B^{\prime}}\left(\mathfrak{M}_{0 \subset B^{\prime}}\right)=\iota(B) \cap \iota\left(B^{\prime}\right) .
$$

However, $\mathfrak{M}_{0 \subset B} \cap \mathfrak{M}_{0 \subset B^{\prime}} \neq 0$ if and only if the segments $B$ and $B^{\prime}$ have a common end-point $a$, and in that case $\mathfrak{M}_{0 \subset B} \cap \mathfrak{M}_{0 \subset B^{\prime}} \neq(a)$. On the other hand, segments $B$ and $B^{\prime}$ have a common end-point $a$ if and only if $\iota(B) \cap \iota\left(B^{\prime}\right)=\iota(a)$. Hence and from lemma 6.2 we infer that

$$
\begin{aligned}
f_{0 \subset B}\left(\mathfrak{M}_{0 \subset B}\right) & \cap f_{0 \subset B^{\prime}}\left(\mathfrak{M}_{0 \subset B^{\prime}}\right) \\
& =f_{0 \subset B}\left(\mathfrak{M}_{0 \subset B} \cap \mathfrak{M}_{0 \cup B^{\prime}}\right)=f_{0 \subset B^{\prime}}\left(\mathfrak{M}_{0 \subset B} \cap \mathfrak{M}_{0 \subset B^{\prime}}\right)=\iota(a),
\end{aligned}
$$

which proves both (i) and (ii).

II. Now if $A \subset B$ and $A^{\prime} \subset B^{\prime}$ are two pairs of $X$ such that $A \neq 0$ $=A^{\prime}$, then, by lemma 5.1, $\mathfrak{M}_{A C B} \cap \mathfrak{M}_{A^{\prime} \subset B^{\prime}} \neq 0$ if and only if $A \subset B \cap B^{\prime}$.

Since $A^{\prime}=0, B^{\prime}$ is a segment of $X$ and so $B \cap B^{\prime}$ is either a vertex or a segment.

If $B \cap B^{\prime}$ is a vertex, then, by $A \subset B \cap B^{\prime}, A$ is also a vertex and $A=B \cap B^{\prime}$. And if $B \cap B^{\prime}$ is a segment, then $A$ is either a vertex or a segment depending on whether $A \neq B \cap B^{\prime}$ or $A=B \cap B^{\prime}$.

a. If $A=B \cap B^{\prime}$, then $\mathfrak{M}_{A \subset B \cap B^{\prime}}=(A)$, and so by lemmas 5.1 and 7.3 (for $A=S$ ) we obtain

$$
\begin{aligned}
f_{A C B}\left(\mathfrak{M}_{A \subset B}\right) & \frown f_{0 \subset B^{\prime}}\left(\mathfrak{M}_{0 \subset B^{\prime}}\right) \\
& =f_{A \subset B}\left(\mathfrak{M}_{A \subset B} \cap \mathfrak{M}_{0 \subset B^{\prime}}\right)=f_{0 \subset B^{\prime}}\left(\mathfrak{M}_{A \subset B} \cap \mathfrak{M}_{0 \subset B^{\prime}}\right)=A^{*},
\end{aligned}
$$

which proves both (i) and (ii) in the case under consideration.

b. And if $A \neq B \cap B^{\prime}$, then $A$ is a vertex of segment $B \cap B^{\prime}$. Since in this case

$$
\mathfrak{M}_{A \subset B \cap B^{\prime}}=\left\{C: O \in C(X), A \in C \subset B \cap B^{\prime}\right\},
$$

then it suffices to put $B \cap B^{\prime}=S$ and to apply lemmas 5.1 and 7.4.

III. Finally, consider the case of $A \neq 0 \neq A^{\prime}$. Here we have, by lemma 5.1, $\mathfrak{M}_{\subset A B} \cap \mathfrak{M}_{A^{\prime} \subset B^{\prime}} \neq 0$ and only if $A \cup A^{\prime} \subset B \cap B^{\prime}$, and by lemma 7.8, $A \cup A^{\prime} \subset B \cap B^{\prime}$ if and only if $f_{A \subset B}\left(\mathfrak{M}_{A \subset B}\right) \cap f_{A^{\prime} \subset B^{\prime}}\left(\mathfrak{M}_{A^{\prime} \subset B^{\prime}}\right)$ $\neq 0$, and in that case, by the same lemmas,

and

$$
\mathfrak{M}_{A \subset B} \cap \mathfrak{M}_{A^{\prime} \subset B^{\prime}}=\mathfrak{M}_{A \cup A^{\prime} \subset B \cup B^{\prime}}
$$

$f_{A C B}\left(\mathfrak{M}_{A C B}\right) \cap f_{A^{\prime} C B^{\prime}}\left(\mathfrak{M}_{A^{\prime} C B^{\prime}}\right)=f_{A C B}\left(\mathfrak{M}_{A \cup A^{\prime} \subset B \cap B^{\prime}}\right)=f_{A^{\prime} \subset B^{\prime}}\left(\mathfrak{M}_{A \cup A^{\prime} \subset B \cap B^{\prime}}\right)$.

Hence, by lemma 7.6, condition (i) holds true and, by lemma 7.8, condition (ii) is also satisfied.

Since $\bigcup_{A \subset B} f_{A \subset B}\left(\mathfrak{M}_{A \subset B}\right)=\alpha(X)$ by 6.1 and 7.1, then an immediate consequence of lemma 2.1 of [1] and lemma 8.1 above is the following theorem:

8.2. The combined function

given by the formula

$$
f: C(X) \rightarrow \alpha(X)
$$

$$
f \mid \mathfrak{M}_{A \subset B}=f_{A \subset B} \quad \text { for all pairs } A \subset B \text { of } X
$$

is a homeomorphism onto.

§ 9. Main result. Recall (cf. [1], $\S 8$ ) that if $P$ is a polyhedron, then by $E_{P}$ we denote the closure of a subset of $P$ consisting of all points $p \in P$ for which there exists a closed neighbourhood topologically equivalent to a disc (i.e., to a 2 -dimensional ball) and such that $p$ lies on the boundary of that disc.

Theorem 8.2 together with some previous results allows us to formulate now the following

Characterization Theorem. Polyhedron $P$ of dimension $\operatorname{dim} P=2$ is the hyperspace if and only if $P$ is a 2-dimensional ball.

If $P$ is a 2-dimensional ball, then $P$ is the hyperspace for an arc and for a simple closed curve.

Polyhedron $P$ of dimension $\operatorname{dim} P>2$ is the hyperspace for an acyclic continuum if and only if its subset $E_{P}$ is homeomorphic to a finite connected and acyelic graph, and $P$ is the a-polyhedron for that graph.

If $E_{P}$ is homeomorphic to a finite connected and acyclic graph $X$ and $P$ is the a-polyhedron $\alpha(X)$ for $X$, then $P$ is the hyperspace $C(X)$ for $X$.

Pro of. Let $\operatorname{dim} P=2$. If $P$ is a hyperspace, then, by theorem 9.1 from [1], $P$ is a 2 -dimensional ball. And conversely, if $P$ is a 2 -dimensional ball, then, by examples 1 and 2 of $\S 3$ from [1], $P$ is the hyperspace for an are and for a simple closed curve.

Let $\operatorname{dim} P>2$. If $P$ is the hyperspace for an acyclic continuum, then, again by theorem 9.1 from [1], it is the hyperspace $C(X)$ for one $X$ only which must be a finite connected and acyclic graph. By theorem 9.2 
from [1], it must also be $X \underset{\text { top }}{=} E_{P}$. Hence $E_{P}$ is homeomorphic to a finite connected and acyclic graph, and so there exists an embedding $\iota: E_{P} \rightarrow I^{N}$, as defined in $\$ 3$ of the present paper. Such an embedding yields the $a$-polyhedron $\alpha\left(E_{P}\right)$ and we have shown the equality $\alpha\left(E_{P}\right) \underset{\text { top }}{=} O\left(E_{P}\right)$ : But, in view of equality $E_{P} \underset{\text { top }}{=} X$, we have $O\left(E_{P}\right) \underset{\text { top }}{=} O(X)$, and since $C(X) \underset{\text { top }}{=} P$ by assumption, then, finally, $\alpha\left(E_{P}\right) \underset{\text { top }}{=} P$. This means that $P$ is the $\alpha$-polyhedron for $E_{P}$.

Conversely, if $E_{P}$ is homeomorphic to a finite connected and acyclic graph $X$ and $P \underset{\text { top }}{=} a(X)$, then the equality $a(X) \underset{\text { top }}{=} C(X)$, proved in 8.2 , implies $P \underset{\text { top }}{=} C(X)$.

§10. Examples. As we know, each finite, connected and acyclic graph $X$ yields $\alpha$-polyhedron $\alpha(X)$ (cf. $\S 3$ ) and we have learned how to represent this polyhedron by its geometric diagram in $E^{3}$ (cf. $\S 4$ ). In view of Characterization Theorem, the geometric diagram of $\alpha(X)$ can: be viewed as a diagram of the hyperspace $C(X)$ itself whenever $\operatorname{dim} C(X)$ $>2$ or, what is the same by virtue of theorem 7.4 from [1], whenever $X$ contains a ramification point (i.e., for $X$ topologically distinct from an: arc). To illustrate this and to show how a complementary procedure can be applied to that diagram in order to discover all particulars of the structure of the respective $C(\bar{X})$ (see especially example 6)-consider now the following examples.

1. Recall first (cf. [1], §3, example 1) that the hyperspace $C(I)$ of a segment $I$ (topologically, of an are) is a 2 -dimensional ball.

2. Consider now a $k$-are, i.e., a union of $k$ segments joined at one vertex (fig. 10).

The polyhedron $C(X)$ is a $k$-dimensional ball to the surface of which $k 2$-dimensional balls are attached along a $k$-arc (for the diagram of $C(X)$, see fig. 11$)$.

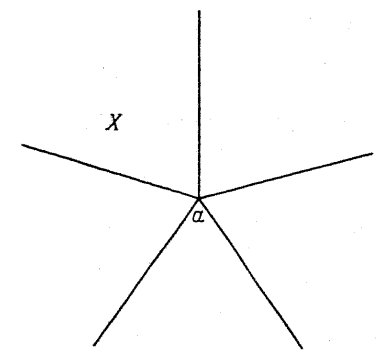

Fig. 10

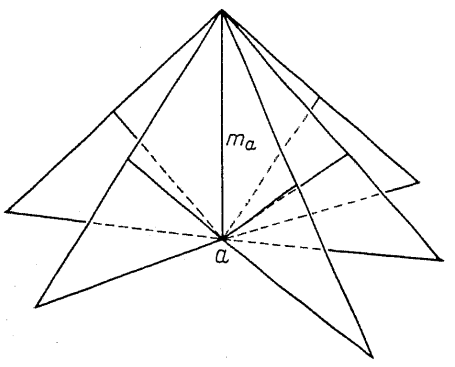

Fig. 11
3. Propositions 6.4 and 7.4, both from [1], imply that the only 3-dimensional polyhedron which is $C(X)$ for $X$ acyclic is a 3 -dimensional ball with three 2 -dimensional balls attached to its surface along a 3 -arc (see fig. 12).

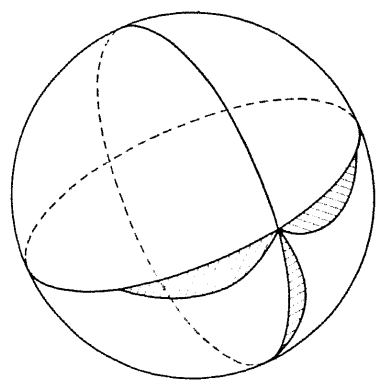

Fig. 12

4. One may also ask about the 4-dimensional polyhedra which are $C(X)$ for $X$ acyclic. It turns out (cf. [1], theorem 7.4) that there are two of them, because there are only two topologically distinct, connected and acyclic graphs possessing 4 end-points (see fig. 13).
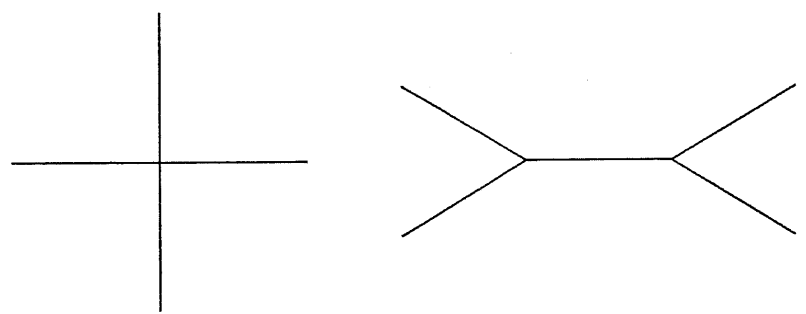

Fig. 13

The hyperspace of the first graph we already know: it is a 4-dimensional ball to the surface of which four 2-dimensional balls are attached along a 4 -arc.

The geometric diagram of the hyperspace of the second graph is given in fig. 14. It is a 4 -dimensional ball (represented by $m \overline{a b}$ ) to the surface of which are attached two 3 -dimensional balls (represented by $m_{a}$ and $m_{b}$ ) along 2 - dimensional balls meeting at one point (representing $\overline{a b}$ ), and to the surface of the union of these two 3 -dimensional balls five 
2-dimensional balls are attached in such a way that their common part with each 3 -dimensional ball is a 3-are and their common part with the 4 -dimensional ball is the point representing $\overline{a b}$.

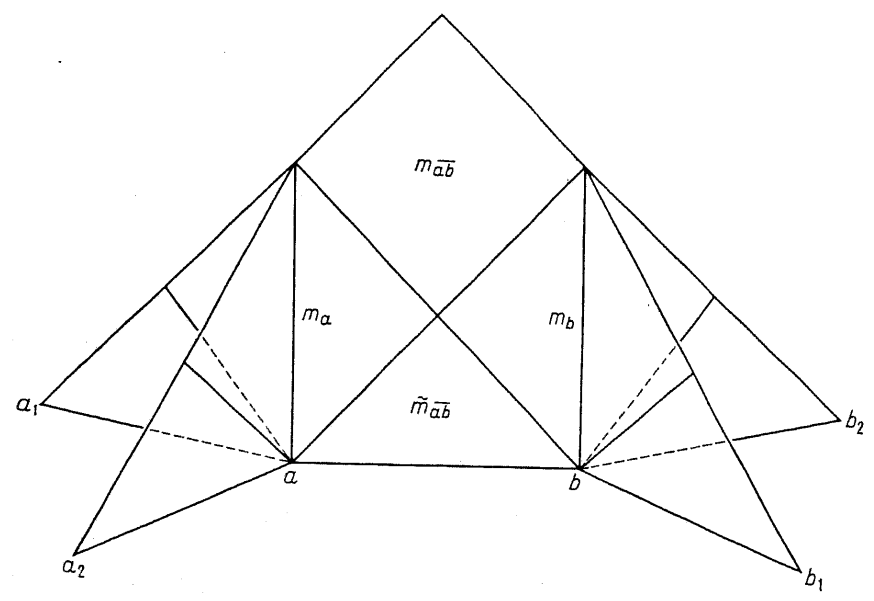

Fig. 14

5. Similarly, since there are three (topologically distinct) connected and acyclic graphs possessing 5 end-points (see fig. 15), there are also only three 5 -dimensional polyhedra which are $O(X)$ for acyclic $X$.
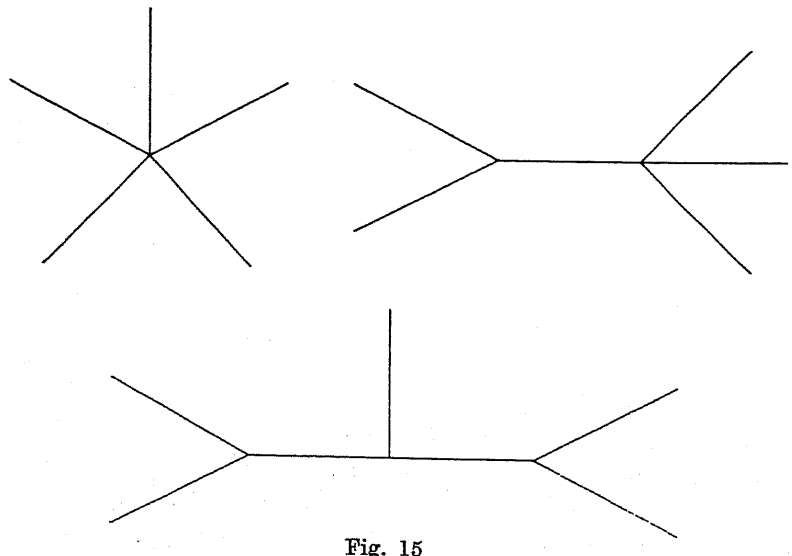

Fig. 15
The geometric diagrams of these three polyhedra, from which their structure can easily be deduced, are given below in fig. 16 .
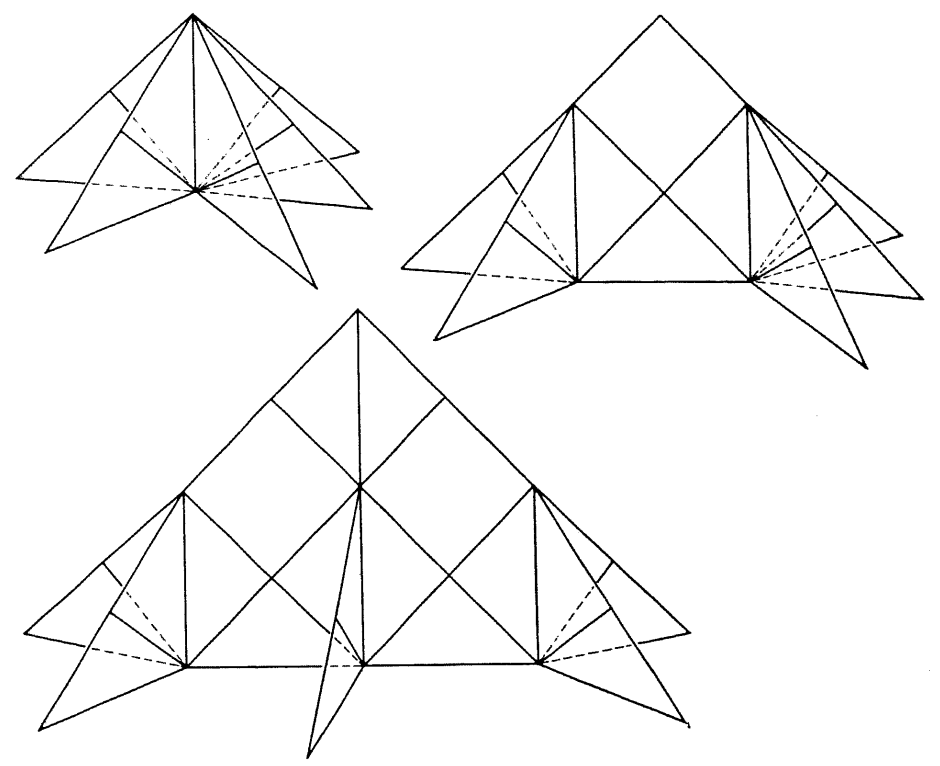

Fig. 16

6. The number of polyhedra of higher dimensions which are $C(X)$ for $X$ acyclic, rapidly increases. For instance, there are seven 6 -dimensional polyhedra of this kind and thirteen 7 -dimensional ones. However, we do not intend to give here atlas of polyhedra $O(X)$ for $X$ acyclic. Instead, in order to provide some idea of the complex structure of the polyhedra involved, we shall consider in some detail the polyhedron $O(X)$ for the graph given in fig. 17 .

The geometric diagram of the 11-dimensional polyhedron $O(X)$ drawn according to the rules described in $\S 4$ is given in fig. 18. In its description (the number standing below or on the left side of $m_{A}$ denotes the dimension of $\left.\dot{M}_{A \subset Q(A, 1)}\right)$ we have used a convenient way of denoting subgraphs of $X$ which we shall now shortly explain. For that purpose let us look at the bunches of triangles which lie over vertex $d$. The lowest $m_{d}$ represents cube $M_{(d) \subset Q(d, 1)}$. The middle one represents cube $M_{A \subset Q(A, 1)}$, where $A$ is an internal subgraph of $X$ of diameter $\delta(A)=2$, 
$d$ being the middle element. Clearly, $A=\overline{d b} \cup \overline{d e}$, which we write as $A=d-b, e$, and so the middle bunch is denoted by $m_{d-b, e}$. Finally;

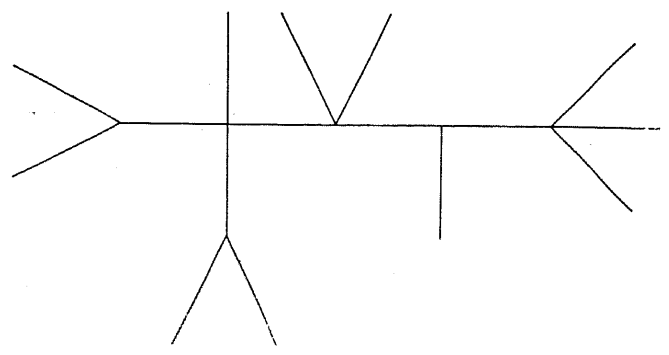

Fig. 17

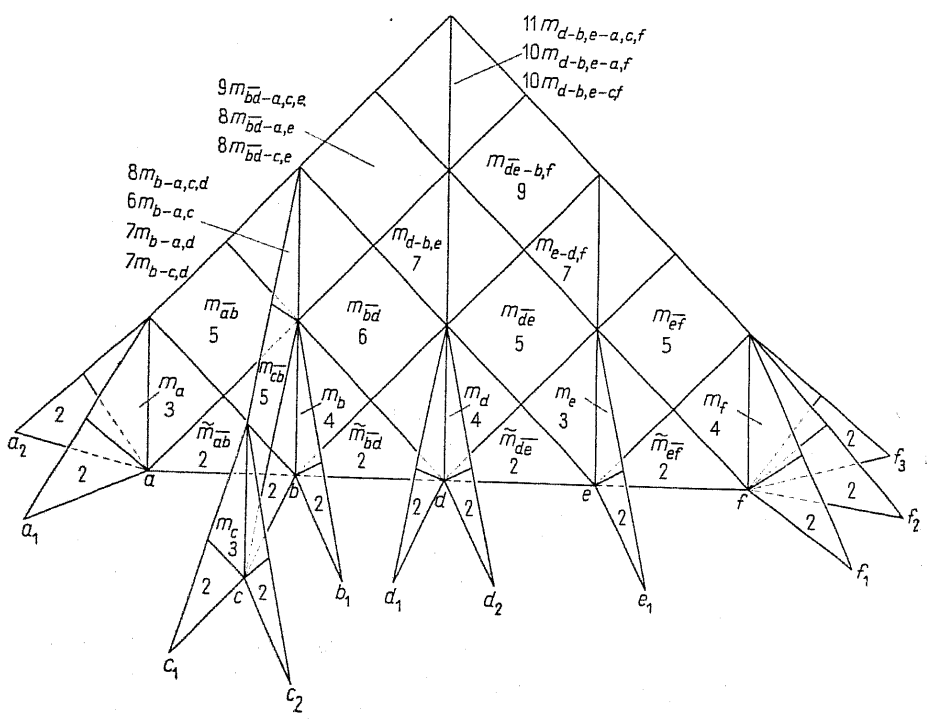

Fig. 18

the upper one represents cubes $M_{A \subset Q(A, 1)}$, where $A$ is an internal subgraph of $X$ with $d$ as its middle element and of diameter $\delta(A)=4$. There are 3 such subgraphs, all obtained by joining to $d-b, e$ some segments, and we denote them by writing down all the vertices which are endpoints of those segments. Thus those 3 subgraphs are denoted by, respectively,

$$
a-b, e-a, f, \quad a-b, e-c, f, \quad a-b, e-a, c, f .
$$

Here we meet for the first time the case where a certain element of the geometric diagram represents more than one cube $M_{A \subset Q(A, 1)}$. In fact, the top square in fig. 18 (a bunch consisting of two triangles) represents cubes $M_{A \subset Q(A, 1)}$ for three distinct subgraphs $A$ of $X$ listed in (1).

Now we shall give another example of a representation by one element of the geometric diagram of several balls from $C(X)$ and proceed to show how to overcome the difficulty.

Consider the ball $M_{(b) \subset Q(b, 1)}$. As we know, this is a 4-dimensional ball given by the analytic formula

$$
M_{(b) \subset Q(b, 1)}=\left|\left(b^{*} ; \overline{b a}\right) \times\left(b^{*} ; \overline{b c}\right) \times\left(b^{*} ; \overline{b b_{1}}\right) \times\left(b^{*} ; \overline{b \bar{d}}\right)\right|,
$$

and it has the face

$$
\left|\left(\frac{1}{2}\right) \times\left(b^{*} ; \overline{b c}\right) \times\left(b^{*} ; \overline{b b_{1}}\right) \times\left(b^{*} ; \overline{b \bar{d}}\right)\right|
$$

in common with the ball $M_{\overline{a b}} \subset Q(\overline{a b}, 1)$, the face

$$
\left|\left(b^{*} ; \overline{b a}\right) \times\left(\frac{1}{2}\right) \times\left(b^{*} ; \overline{b b_{1}}\right) \times\left(b^{*} ; \overline{b \bar{d}}\right)\right|
$$

in common with the ball $M_{\overline{b c} \subset Q(\overline{b c}, 1)}$, the face

$$
\left|\left(b^{*} ; \overline{b a}\right) \times\left(b^{*} ; \overline{b c}\right) \times\left(b^{*} ; \overline{b b_{1}}\right) \times\left(\frac{1}{2}\right)\right|
$$

in common with the ball $M_{\overline{b d} \subset Q(\overline{b d}, 1)}$ (in all cases, $\left(\frac{1}{2}\right)$ instead of the vector $\left(b^{*} ; \overline{v w}\right)$ denotes the coordinate $\frac{1}{2}$ on the axis determined by this vector). Hence the balls $M_{\overline{a b} \subset Q(\overline{a d}, 1)}, M_{\overline{b c} \subset Q(\overline{b c}, 1)}$, and $M_{\bar{b} \bar{c} \subset Q(\bar{b}, 1)}$ have the common edge

$$
E=\left|\left(\frac{1}{2}\right) \times\left(\frac{1}{2}\right) \times\left(b^{*} ; \overline{b b_{1}}\right) \times\left(\frac{1}{2}\right)\right|,
$$

and any two of them meet along a 2 -dimensional face stretched upon edge $E$ and the "missing" edge. For instance, $M_{\overline{a b} \subset Q(\overline{a b}, 1)}$ and $M_{\overline{b c} \subset Q(\overline{b c}, 1)}$ meet along the face

$$
\left|\left(\frac{1}{2}\right) \times\left(\frac{1}{2}\right) \times\left(b^{*} ; \overline{b b_{1}}\right) \times\left(b^{*} ; \overline{b d}\right)\right| .
$$

If we try to present this situation in a drawing it may look somewhat like fig. 19. Note that in our geometric diagram of $C(X)$ (see fig. 18) edge $E$ and the three 2 -dimensional faces common to the pairs of balls $M_{\overline{a b} \subset Q(\overline{a b}, 1)}, M_{\overline{b c} \subset Q(\overline{b c}, 1)}$ and $M_{\overline{b d} \subset Q\left(\bar{b} \bar{d}_{11}\right)}$ are represented by a single point. 


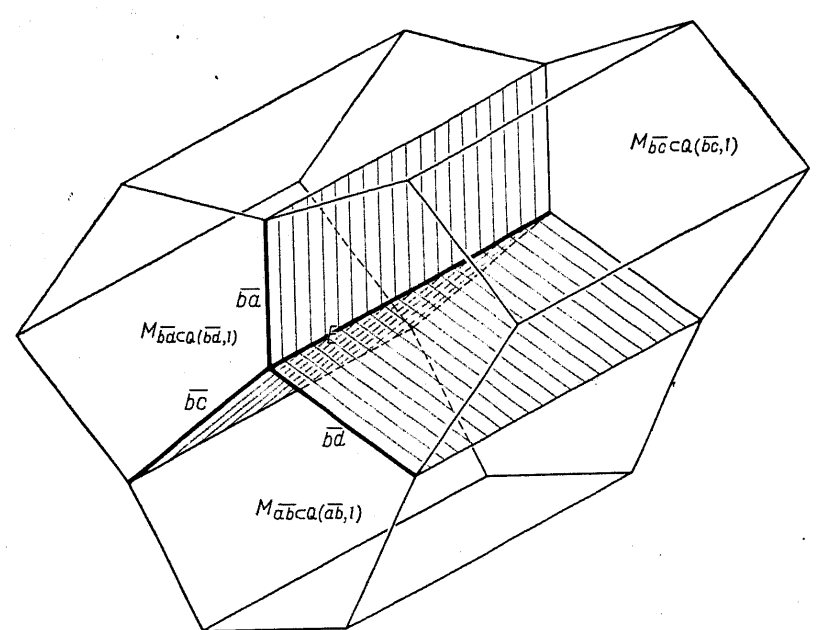

Fig. 19

Now, the 4-dimensional ball $M_{(b) \subset Q(b, 1)}$ (which is not represented in fig. 19; one can imagine it as lying under the drawing and glued to each of the balls $M_{\overline{a b} \subset Q(\overline{a b}, 1)}, M_{\overline{b c} \subset Q(\bar{b} c, 1)}$ and $M_{\overline{b a d} \subset Q(\bar{b} d, 1)}$ along the respective 3 -dimensional face) has 16 vertices. From four of them issue four balls $M_{A C Q(A, 1)}$ represented in fig. 18 by the bunch of three triangles lying over $m_{b}$ : from $\left(\frac{1}{2}, \frac{1}{2}, 0,0\right)$ issues $M_{b-a, c \subset Q((b-a, c), 1)}$, from $\left(\frac{1}{2}, 0,0, \frac{1}{2}\right)$ issues $M_{b-a, d \subset Q((b-a, d), 1)}$, from $\left(0, \frac{1}{2}, 0, \frac{1}{2}\right)$ issues $M_{b-c, d \subset Q(b-c, d), 1}$, and from $\left(\frac{1}{2}, \frac{1}{2}, 0,0\right)$ issues $M_{b-a, c, d \subset Q(b-a, c, d), 1)}$. Here we have another example of a representation by one element of a geometric diagram of several balls from $C(X)$.

If we write down analytical formulas for the four balls $M_{b-a, c, d \subset((b-a, c, d), 1)}, M_{b-a, c \subset Q(b-a, c), 1)}, M_{b-a, d \subset Q((b-a, d), 1)}$ and $M_{b-c, d \subset Q(b-c, d), 1)}$, then it is easy to discover their mutual relations. For instance, all the four balls except the second meet the ball $M_{d-b, e \subset Q(d-b, e), 1)}$ : the first along the 3 -dimensional face

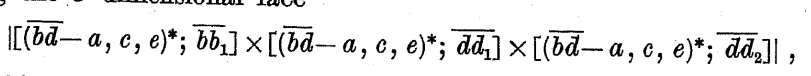

the third along the 4 -dimensional face

$\left\lfloor\left[(\overline{b \bar{d}}-a, e)^{*} ; \overline{b c}\right] \times\left[(\overline{b \bar{d}}-a, e)^{*} ; \overline{b b_{1}}\right] \times\left[(\overline{b \bar{d}}-a, e)^{*} ; \overline{d \bar{d}}_{1}\right] \times\left[(\bar{b} \bar{d}-c, e)^{*} ; \bar{d}_{d_{2}}\right] \mid\right.$, and the fourth along the 4 -dimensional face

$\left|\left[[\overline{(\bar{b} d}-c, e)^{*} ; \overline{b a}\right] \times\left[(\overline{b \bar{d}}-c, e)^{*} ; \overline{b b}_{1}\right] \times\left[(\overline{b \bar{d}}-c, e)^{*} ; \overline{d \bar{d}}_{1}\right] \times\left[(\overline{b \bar{d}}-e, e)^{*} ; \bar{d}_{2}\right]\right|$.
Similarly, the first, third, and fourth balls of the four listed above meet any of the three balls -

$M_{\bar{b} \bar{d}-a, c, e \subset Q(\bar{b} d-a, c, e), 1)}, \quad M_{\bar{b} d-a, e \subset Q((\bar{b} \bar{d}-a, e), 1)}, \quad$ and $\quad M_{\overline{b d}-c, e \subset Q((\bar{b} \bar{d}-c, e), 1)}$.

We shall not pursue this analysis further since it can be completed by anyone. Instead, let us mention two more exemplary features of our geometric diagram of $C(X)$ : first, using the geometric diagram and, if necessary, the analytical apparatus of coordinates one can find a neighbourhood of any $C \epsilon C(X)$, and secondly, such a geometric diagram of $C(X)$ contains also diagrams of all subgraphs of $X$. For instance, if we remove from $X$ in fig. 17 three segments, $\overline{f f}_{1}, \overline{f f}_{2}$, and $\overline{f f}_{3}$, then the diagram of the remaining subgraph can be obtained from the diagram of the whole $C(X)$ by removing the strip on the right-hand side of fig. 18 consisting of all $\widetilde{m}$ and $m_{A}$ such that the letter $f$ appears in $A$ (with the exception of $\tilde{m}_{\overline{e f}}$, of course, but it does not belong to the strip).

Looking upon the geometric diagram of $C(X)$ as it gradually emerges. from the partial diagrams of $\alpha^{\prime}(X), \alpha^{\prime}(X) \cup a_{0}(X), \alpha^{\prime}(X) \cup a_{0}(X) \cup \alpha_{1}(X), \ldots$ one may see in it a kind of a pyramid whose base is a 1 -dimensional curve consisting of all one-point subcontinua of $X$. $\varrho(X)$ in notation of [2]). To this base is first attached a set of perpendicular triangles. This is $\alpha^{\prime}(X)$. To form $\alpha^{\prime}(X) \cup \alpha_{0}(X)$ we add balls to $\alpha^{\prime}(X)$, each ball of dimension 3 or more. It can be said that these balls "fill up the hollows" of $\alpha^{\prime}(X)$. And so on: in each subsequent step we "fill up the hollows" of the preceding one with balls of still higher dimension (by virtue of lemmas 2.1 and 7.2 each ball of $\alpha_{k}(X)$ has dimension $k+3$ or more: and by lemmas 2.2 and 7.2 , its dimension is in any case greater than that of any ball in the "hollow"), the set of these added balls becoming more and more "narrow" (although their number may occasionally increase) and more and more "thick" (the latter thanks to the increase of dimension), aiming at the top of the pyramid formed in every case by the ball of the highest dimension with the point representing $X$ as one of its free. vertices.

\section{References}

[1] R. Duda, On the hyperspace of subcontinua of a finite graph, I, Fund. Math. 62: (1968), pp. 265-286.

[2] J. L. Kelley, Hyperspaces of a continuum, Trans. Amer. Math. Soc. 52 (1942), pp. 22-36.

[3] C. Kuratowski, Topologie, two volumes, Warszawa-Wrocław 1948.

INSTitute OF MATHEMATICS OF THE POLISH ACADEMY OF SCIENCES

Regu par la Rédaction le 10. 4. 1967 